\title{
Analytical modelling of multiservice switching networks with multiservice sources and resource management mechanisms
}

\author{
Mariusz Głąbowski $^{1}$ (D) - Maciej Sobieraj ${ }^{1}$
}

Published online: 16 March 2017

(C) The Author(s) 2017. This article is published with open access at Springerlink.com

\begin{abstract}
This article proposes a new analytical method of determining occupancy distribution and blocking probability in multiservice switching networks with admission control mechanisms and resource management mechanisms. The proposed method is a universal one, which allows for determining blocking probability using the same model, regardless of the type of admission control mechanisms and resource management mechanisms. The versatility of the method is demonstrated in the article for two selected mechanisms, i.e. a threshold mechanism and a resource reservation mechanisms. The switching networks under consideration are offered multiservice traffic streams, generated by the so-called multiservice sources, according to Erlang, Engset and Pascal distributions. The proposed method is based on a generalised way of determining traffic characteristics for inter-stage links of switching networks with resource and call management mechanisms. Particular attention is given to determining the influence of the introduced mechanisms in outgoing links of the switching network on the traffic properties of inter-stage links of the network. The results of analytical calculations are then compared with the results obtained in digital simulation for a number of selected switching networks.
\end{abstract}

Keywords Switching networks - Multiservice traffic . Blocking probability

Mariusz Głąbowski

mariusz.glabowski@put.poznan.pl

Maciej Sobieraj

maciej.sobieraj@put.poznan.pl

1 Faculty of Electronics and Telecommunications, Poznan University of Technology, ul. Polanka 3, 60-965 Poznan, Poland

\section{Introduction}

The types of services in modern telecommunication networks can be grouped into three types of traffic, i.e. streaming, adaptive and elastic traffic [1], with the possibility of a change in resources (usually bit rate) allocated to service a call of a given traffic class, and also a change in the service time of this call as a criterion for their differentiation. In services that generate streaming traffic, the demanded level of resources is fixed and cannot undergo any changes without simultaneous increase in the load of the system. Adaptive traffic allows a decrease in the size of allocated resources for new calls, with their service time being retained. Traffic of this kind is typically related to real time services, for example video transmission, in the case of which any prolongation of the transmission time is not acceptable. With elastic traffic, the important thing is-regardless of available resourcesto execute a given service (e.g., transmission of all data). For this purpose, along with an increase in the load of network resources, it is possible to decrease the size of resources allocated to new calls with simultaneous prolongation of their service time. Traffic of this type is used in a wide spectrum of services that make use of the Transmission Control Protocol (TCP) as a transmission protocol.

With the ever-growing demand for network operators to increase available bit rates and throughput, the development of both transmission networks and increase in the efficiency of nodes of a network have gathered particular momentum. In the case of transmission networks, particular technologies have been developed that make it possible to effectively service traffic streams with very different demands in terms of their bit rates, service time and the required Quality of Service parameters. In order to service traffic streams with demands ranging from kilobits per second to gigabits per second more effectively, the literature proposes a num- 
ber of technologies, including the Optical Burst Switching (OBS) [2], Optical Burst Transport Network (OBTN) [3] and optical elastic networks (EON) [4,5], allowing operators to manage available wavelengths (optical spectrum) more effectively.

The other factor, apart from the transmission network, that directly influences the operation of telecommunication networks, regardless of the applied transmission medium, is network nodes and their switches and routers. Each of these devices includes a switching network that is executed according to a given switching structure. The studies that have been carried out, both those that focused on theoretical aspects [6-10], and those related to the execution of large optical nodes [11,12], indicate that one of the most effective switching structures that ensures high scalability is the multistage Clos network. A multi-stage Clos network, both optical and electronic, allows nodes with large capacities (with a large number of inputs and outputs) to be constructed using switching elements with small capacities. This is particularly important in the case of optical networks in which, due to technological constraints, switching elements used in constructing multi-stage switching networks have relatively low numbers of inputs and outputs. One of the first large-scale routers implemented on the basis of high-speed optical switch elements, was described in [11]. This solution, in which a three-stage Clos network is used, clearly indicates the indisputable advantage of multiservice networks over single-stage systems. The universal structure of a Clos network also makes it applicable in large data centers [13]. The considered structure of a Clos switching network is a blocking structure. In the blocking switching networks, contrary to the non-blocking structures, the phenomenon of internal blocking, manifested by the lack of any possibility of setting up a connection between a free input and a free output, exists [14]. However, the use of nonblocking networks is not financially viable as it leads to a situation in which a potential increase in network effectiveness is not matched economically by relevant financial input necessitated by its construction (a large number of stages and switching elements) [15-17].

Electronic multi-stage switching networks and optical multi-stage switching networks, despite a number of certain differences (e.g., optical transient signal suppression while switching and crosstalk) show many similarities that make their modelling at the call level possible ${ }^{1}$ with the application of the same analytical models [20,21].

This article proposes a new, generalised method for analytical determination of traffic characteristics of multi-stage switching networks servicing traffic streams undergoing

\footnotetext{
1 The analysis of traffic sources with variable bit rate at the packet level can be reduced in its essence to the analysis at the call level (otherwise known as flow level, session level) using, e.g., the concept of equivalent bandwidth [18,19].
}

admission control mechanisms and resource allocation control. These mechanisms are executed by reservation algorithms and threshold algorithms and make it possible to shape the quality policy (e.g., differentiation of access to limited resources [22]), improve the effectiveness of resource usage (e.g., spectrum efficiency and energy efficiency [23]) and increase the stability of operation of network devices (e.g., load balancing [24]). At the same time, the mechanisms allow streaming, elastic and adaptive services to be performed [1]. The proposed approach of the unified modelling scheme for different call admission mechanisms and resource allocation control mechanisms used in switching networks makes it possible to improve the accuracy of the determination of the influence of these mechanisms on the accuracy in the determination of traffic characteristics of switching networks, including in particular on the load of inter-stage links. This method allows us to apply the same calculation model to determine the blocking probability regardless of the resource or call management mechanism used. As a result, the area of application of the method is broad. The general approach to modelling multi-stage switching networks with Clos structure thus developed also permits us to take into consideration traffic streams generated by multiservice Erlang, Engset and Pascal sources [25].

The remaining part of the paper is organised as follows. Section 2 presents the current state of research on the analytical modelling of switching networks with multiservice traffic and resource and call management mechanisms. In Sect. 3, the traffic streams, generated by multiservice sources, are presented, along with a description of the structure of 3-stage switching networks. Section 4 discusses the analytical models of outgoing links of a switching network, while in Sect. 5, a new general model of interstage links of the switching network under consideration is proposed. Section 6 describes the effective availability method for point-to-point and point-togroup blocking probability calculation the switching network with multiservice sources. The results of analytical modelling are compared with simulation data in Sect. 7. Section 8 concludes the paper.

\section{Related works}

Analytical methods of determining the traffic properties of switching networks with multiservice traffic can be divided into two groups. One group of methods is aimed at searching for time-effective methods of solving linear equations resulting from the Markov process that occurs in a communication system. Despite their high accuracy, any practical application of these methods, based on the solution of state equations, is very complicated or even impractical on account of too high a number of states of a communication system involved. An attempt to analyse two-stage switching networks with mul- 
tiservice traffic with the state equations method is presented in [26].

The methods that belong to the other group are based on an approximation of a multidimensional Markov process occurring in switching systems under consideration by a onedimensional Markov chain that is characterised by a product form solution [27,28]. This group of methods of calculating blocking probabilities in switching networks with multiservice traffic coincides with methods presented, for example in [29], that are based on the assumption, proposed by Jacobeus $[30,31]$, of the independent nature of occupancy distributions of links between successive stages of the network. However, the error resulting from the above assumption, proportional to the number of stages in a switching network, as well as high computational complexity prevent these methods from being effectively applied in networks with the number of stages higher than three.

In this group of methods, the most effective ones for modelling switching networks are effective availability methods [32]. The essence and basic assumption in this group of methods is the idea of determining the blocking probability in a multi-stage network on the basis of an equivalent single-stage system, i.e. a non-full availability group (EIG). A parameter that allows calculations in a non-full availability group to be performed is the so-called effective availability. Effective availability can be determined in the multi-stage network in a way that ensures equality of the blocking probability in both multi-stage and single-stage networks [33]. Studies on the application of effective availability methods were initially carried out for networks with single-channel traffic [33-36]. Then, the focus was turned to networks with multiservice traffic [32,37-39].

Initially, research studies dealt with switching networks without taking into account any mechanisms that would differentiate the service quality for individual call streams as a result of, for example, an introduction of call admission control mechanisms (CAC functions) or resource allocation mechanisms. The first analytical methods that allowed appropriate reservation algorithms in particular stages of a switching network to be taken into consideration in calculations of the blocking probability were proposed in, e.g. [40]. The following works dealt with switching networks in which threshold mechanisms had been introduced [41]. Investigations in which the execution of multicast connections in switching networks could be taken into account were also carried out [42].

Despite intense sustained works on the analytical modelling of switching networks with multiservice traffic, most models still apply to switching networks in which multiservice traffic streams are generated by an infinite number of traffic sources (PCT1 streams-Pure Chance Traffic of Type 1, also known as Erlang traffic [43]). However, in presentday networks, the ratio of the number of traffic sources to the capacity of a system is often limited and the value of traffic offered by calls of individual classes depends on the count of traffic sources for individual service classes $[44,45]$.

Studies on modelling switching networks with multiservice traffic generated by a finite number of traffic sources (Engset and Pascal streams) can be divided into two groups, depending on the source type. The literature on the subject distinguishes multiservice systems with single-service traffic sources and multiservice systems with multiservice traffic sources [46]. In the case of the former type of a multiservice system, a single source of a given traffic class can generate one, strictly defined, traffic stream. In the case of multiservice systems with multiservice traffic sources, in turn, a single source can generate different traffic streams that correspond to the classes related to the given source. The first attempts to apply the effective availability method to multiservice traffic sources with single-service Engset and Pascal traffic sources were presented in $[47,48]$. Over time, these have been extended to include multiservice switching networks with single-service Engset traffic sources and derived resource access control mechanisms such as, for example, threshold mechanisms [49].

The authors in [46] attempt to develop a new model of multiservice switching network in which a single source can generate different traffic streams that correspond to service classes related to the given source. In the model considered in the study, traffic sources correspond to real terminals (end devices) that support different types of services (e.g. voice services, video-conferences or data transmission). Thus defined systems are then compared with systems that have earlier been considered in the literature on the subject: multiservice systems with single-service traffic sources, i.e. terminals related to only one type of service. Relevant investigations, originally initiated in [46], are then continued in [50,51]. Głabowski and Sobieraj [50] proposes the PGB$\mathrm{R}$ method for calculations of the point-to-group blocking probability in a switching network with multiservice traffic sources and a reservation algorithm, while [51] discusses the PGB-T method for the determination of point-to-group blocking probability in a network with multiservice traffic sources and threshold mechanisms.

In all hitherto published works, particular resource allocation control mechanisms (threshold mechanisms, reservation mechanisms) required specialized methods of network modelling to be worked out. The methods of modelling networks with reservation mechanisms [50] were developed differently than those for networks with threshold mechanisms [51]. The influence of these mechanisms, applied to output links, on the traffic properties of inter-stage links was determined by scaling the reservation/threshold boundaries in inter-stage links, where the scale coefficient was defined as the ratio between the capacity of output links and inter-stage links. Thus constructed heuristics suffered, however, from a major drawback 

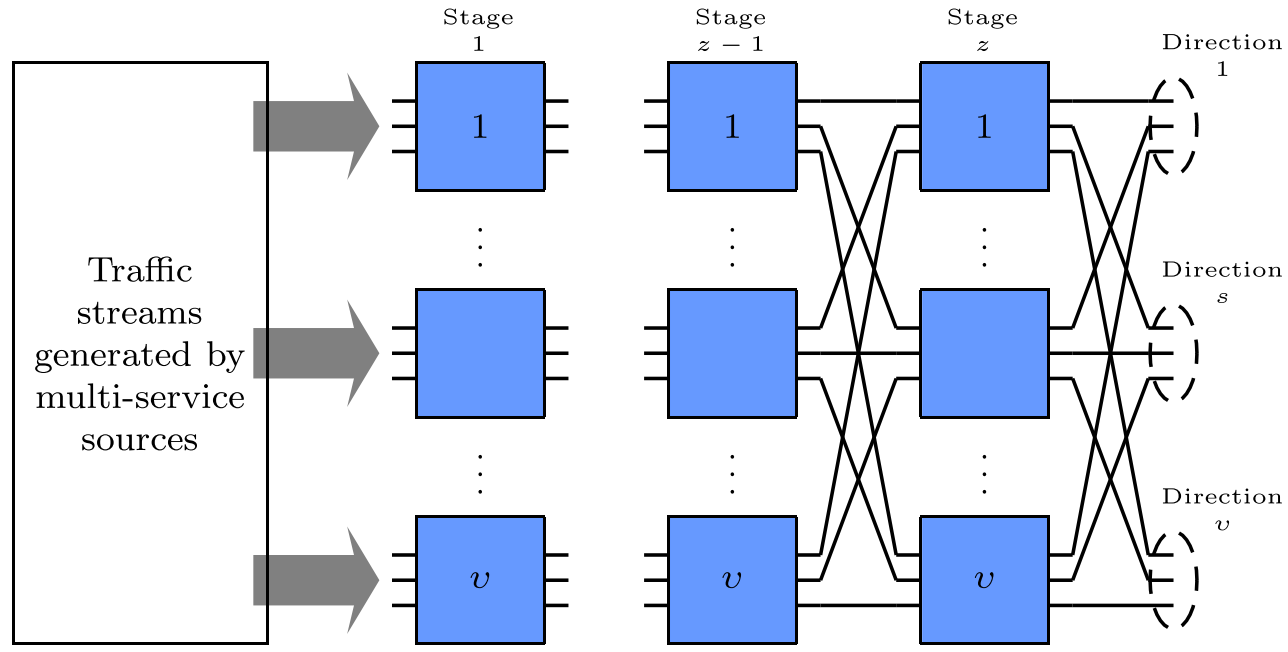

Fig. 1 Model of a multi-stage switching network

resulting in the introduction of certain inaccuracies in determining internal blocking probability.

The present article proposes a new generalised method of analytical determination of traffic characteristics of multistage switching networks with implemented call admission and resource allocation control mechanisms. The proposed approach to modelling different call admission and resource allocation control mechanisms used in switching networks will make it possible to significantly increase the accuracy of determining traffic characteristics of switching networks and, additionally, to take into account traffic streams generated by multiservice Erlang, Engset and Pascal traffic sources [51]. The recurrent method of modelling multiservice switching networks with multiservice sources developed in the article will enable a simultaneous determination of point-to-point and point-to-group blocking probabilities.

\section{General model of a switching network}

\subsection{Structure of offered traffic}

Consider the multiservice $z$-stage switching network [46] with Clos structure presented in Fig. 1. Our assumption is that each inter-stage link has the capacity equal to $f$ AUs (allocation units, e.g., links, channels, basic bandwidth units [52]). The output links of the switching network are grouped into link groups that create the so-called output directions towards the neighbouring nodes of the network. A typical structure of output directions is presented in Fig. 1: each direction includes one output link from each switch of the last stage.

The assumption is that the network is offered calls related to $m$ service classes that belong to set $\mathbb{M}=\{1,2, \ldots, m\}$. A given class $c$ is unequivocally defined by the number $t_{c}$ of AUs necessary to set up a new connection of class $c$, as well as parameter $\mu_{c}$ of the exponential service time distribution for calls of class $c\left(\mu_{c}^{-1}\right.$ - the average service time of a call of class $c$ ). Calls of particular service classes are generated by Erlang, Engset and Pascal traffic sources. Because of the multiservice nature of the traffic sources (the possibility to generate calls of a number of services by just one source), they are grouped into appropriate sets [46]. Another assumption is that each set includes a defined number of traffic classes that generate traffic streams of the same type (i.e. Erlang, Engset and Pascal). The number of generated different traffic streams depends on the set of available services for a given set of traffic sources-for each set of traffic sources a correlated set of available service classes, that is a subset of set $\mathbb{M}$, has been defined. In the system, $s_{I}$ sets of traffic sources that generate Erlang traffic streams have been defined, as well as $s_{J}$ sets of traffic sources that generate Engset traffic streams, and $s_{K}$ sets of traffic sources that generate Pascal traffic streams. The total number of sets of traffic sources in the system is $s=s_{I}+s_{J}+s_{K}$. Symbol $\mathbb{Z}_{\mathrm{En}, j}$ denotes set number $j$ that includes $N_{\mathrm{En}, j}$ Engset sources, symbol $\mathbb{Z}_{\mathrm{Pa}, k}$ denotes set number $k$ that includes $S_{\mathrm{Pa}, k}$ Pascal sources, whereas symbol $\mathbb{Z}_{\mathrm{Er}, i}$ denotes set $i$ of Erlang traffic sources. Sources that belong to set $\mathbb{Z}_{\mathrm{Er}, i}$ can generate call streams from set $\mathbb{C}_{\mathrm{Er}, i}=\left\{1,2, \ldots, c_{\mathrm{Er}, i}\right\}$, where $c_{\mathrm{Er}, i}$ is equal to the number of services available to traffic sources that belong to set $\mathbb{Z}_{\mathrm{Er}, i}$. Similarly, sources that belong to set $\mathbb{Z}_{\mathrm{En}, j}$ can generate call streams that are related to the set of services $\mathbb{C}_{\mathrm{En}, j}=\left\{1,2, \ldots, c_{\mathrm{En}, j}\right\}$, while sources that belong to set $\mathbb{Z}_{\mathrm{Pa}, k}$ can generate call streams that are related to set $\mathbb{C}_{\mathrm{Pa}, k}=\left\{1,2, \ldots, c_{\mathrm{Pa}, k}\right\}$ of available services.

The participation of class $c(c=1,2, \ldots, m)$ in the structure of traffic generated by sources from sets $\mathbb{Z}_{\mathrm{Er}, i}, \mathbb{Z}_{\mathrm{En}, j}$, $\mathbb{Z}_{\mathrm{Pa}, k}$, respectively, is determined by parameters $\eta_{\mathrm{Er}, i, c}$, $\eta_{\mathrm{En}, j, c} \eta_{\mathrm{Pa}, k, c}$. These parameters for individual sets of Erlang, 
Engset and Pascal traffic sources satisfy the following dependencies:

$$
\begin{aligned}
& \forall_{i=1, \ldots, s_{I}} \sum_{c=1}^{c_{\mathrm{E}, i}} \eta_{\mathrm{Er}, i, c}=1, \\
& \forall_{j=1, \ldots, s_{J}} \sum_{c=1}^{c_{\mathrm{En}, j}} \eta_{\mathrm{En}, j, c}=1, \\
& \forall_{k=1, \ldots, s_{K}} \sum_{c=1}^{c_{\mathrm{Pa}, k}} \eta_{\mathrm{Pa}, k, c}=1 .
\end{aligned}
$$

In the case of Erlang traffic streams, the intensity of arrival of new calls does not depend on the number of serviced traffic sources (the model assumes an infinite number of traffic sources) $[25,53]$. As a result, for the considered system, the average value of traffic generated by Erlang sources from set $\mathbb{Z}_{\mathrm{Er}, i}$ generating calls of class $c$ is:

$$
A_{\mathrm{Er}, i, c}=\eta_{\mathrm{Er}, i, c} \lambda_{\mathrm{Er}, i} / \mu_{c},
$$

where $\lambda_{\mathrm{Er}, i}$ is the intensity of arrival of calls generated by sources that belong to set $\mathbb{Z}_{\mathrm{Er}, i}$.

In the case of Engset sources, the intensity of arrival of new calls of particular classes decreases with the increase in the number of serviced traffic sources (the occupancy state of the system), whereas in the case of Pascal sources, the intensity of arrival of new calls of particular classes increases along with the increase in the number of serviced traffic sources (the occupancy state of the system) [25,53]. Values $A_{\mathrm{En}, j, c}(n)$ and $A_{\mathrm{Pa}, k, c}(n)$ of traffic offered, respectively, by Engset sources from set $\mathbb{Z}_{\mathrm{En}, j}$ and Pascal sources from set $\mathbb{Z}_{\mathrm{Pa}, k}$, generating calls of class $c$ in state $n$ of busy AUs, can then be determined as follows:

$$
\begin{aligned}
& A_{\mathrm{En}, j, c}(n)=\left[\eta_{\mathrm{En}, j, c} N_{\mathrm{En}, j}-y_{\mathrm{En}, j, c}(n)\right] \alpha_{\mathrm{En}, j}, \\
& A_{\mathrm{Pa}, k, c}(n)=\left[\eta_{\mathrm{Pa}, k, c} S_{\mathrm{Pa}, k}+y_{\mathrm{Pa}, k, c}(n)\right] \beta_{\mathrm{Pa}, k},
\end{aligned}
$$

where

- $y_{\mathrm{En}, j, c}(n)$ - the average number of calls of class $c$ that are generated by Engset sources from set $\mathbb{Z}_{\mathrm{En}, j}$ and serviced in the system in the occupancy state of $n$ AUs,

- $y_{\mathrm{Pa}, k, c}(n)$ - the average number of calls of class $c$ that are generated by Pascal sources from set $\mathbb{Z}_{\mathrm{Pa}, k}$ and serviced in the system in the occupancy state of $n$ AUs,

$-\alpha_{\mathrm{En}, j}$ - the average intensity of traffic offered by a single Engset source from set $\mathbb{Z}_{\mathrm{En}, j}$ :

$$
\alpha_{\mathrm{En}, j}=\sum_{c=1}^{c_{\mathrm{En}, j}} \eta_{\mathrm{En}, j, c} \gamma_{\mathrm{En}, j} / \mu_{c},
$$

where $\gamma_{\mathrm{En}, j}$-intensity of generating calls by a single free Engset source that belongs to set $\mathbb{Z}_{\mathrm{En}, j}$,

$-\beta_{\mathrm{Pa}, k}$ - the average intensity of traffic offered by a single Pascal source that belongs to set $\mathbb{Z}_{\mathrm{Pa}, k}$ :

$$
\beta_{\mathrm{Pa}, k}=\sum_{c=1}^{c_{\mathrm{Pa}, k}} \eta_{\mathrm{Pa}, k, c} \gamma_{\mathrm{Pa}, k} / \mu_{c},
$$

where $\gamma_{\mathrm{Pa}, k}$-intensity of call generation by a single free Pascal source that belongs to set $\mathbb{Z}_{\mathrm{Pa}, k}$.

We can observe that with these three types of traffic streams (Erlang, Engset, Pascal), we are able to describe all dependencies between call intensity and state of the system (the number of active sources of particular classes). In the case of Erlang traffic stream, when the load of the system increases or decreases, the call intensity is unchanged. In the case of Engset traffic streams, when the load of the system increases, the call intensity decreases. In the case of Pascal traffic streams, when the load of the system increases, the call intensity also increases.

\subsection{Algorithms for setting up connections in a switching network}

Switching networks can operate with either of the two types of selection, i.e. point-to-point selection or point-to-group selection. Let us consider the point-to-group algorithm first. Setting up a connection in the switching network with multiservice traffic with this type of selection commences with the determination of a switch of the first stage where, at the input link, a call of a given class $c$ arrives. Then, a switch of the last stage that has an output link in the demanded direction with at least $t_{c}$ free AUs is chosen according to a randomized algorithm. For thus determined switches, i.e. input and output switches, the algorithm attempts to set up a connection within the network. The existence of a free connecting path is followed by the execution of this connection. Otherwise, another attempt to set up a connection will be made, i.e. the controlling device will attempt to find a free connecting path between a given switch of the first stage and a newly determined switch of the last stage that has at least $t_{c}$ free AUs in the demanded direction. If this connecting path exists, then the connection will be executed; if not, the third attempt to set up a connection will be made according to the same procedure. If the connection cannot be successfully executed during the last possible attempt no. $v$ ( $v$ is the number of switches of the last stage and, at the same time, the number of links of the output group), then the call will be lost due to internal blocking in the switching network. If, in a given state of the switching network, none of the switches of the last stage that would have output links with 
Fig. 2 Model of a limited-availability group with reservation

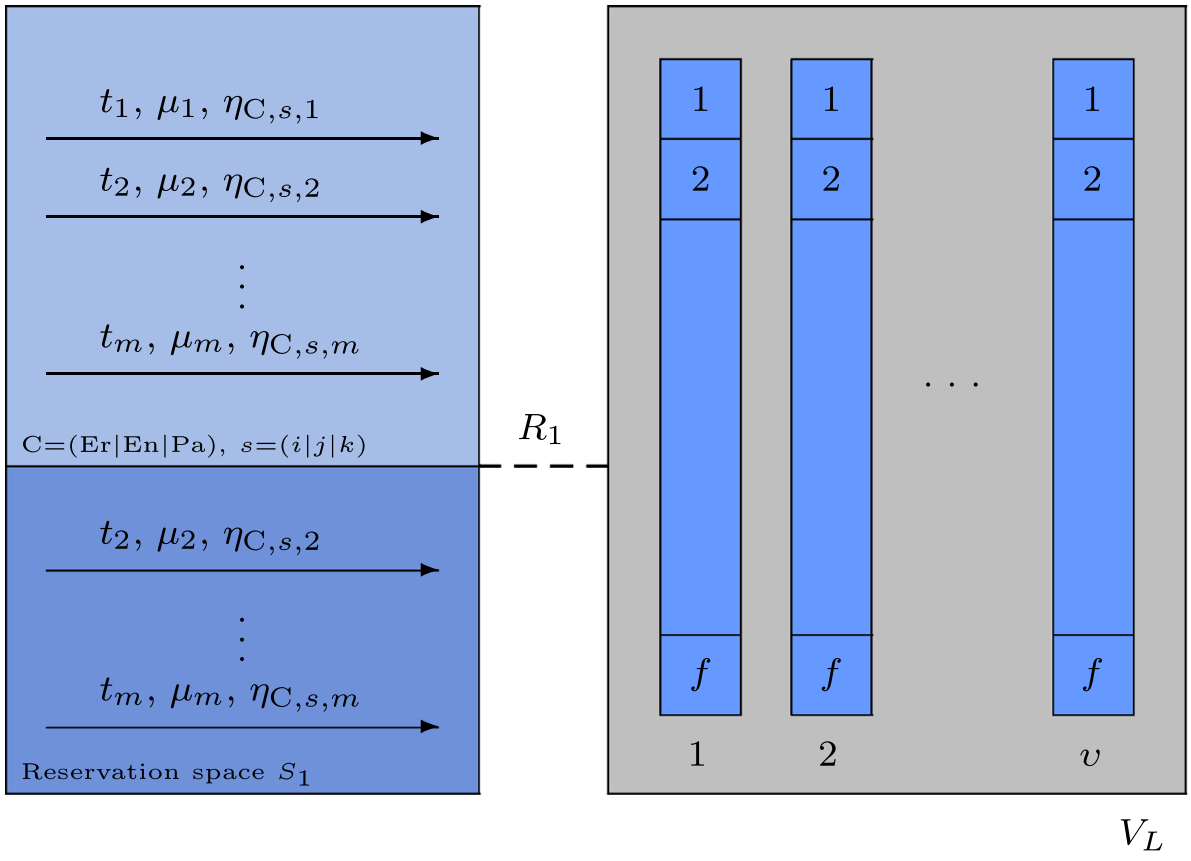

at least $t_{c}$ free AUs are available, then the call will be lost due to external blocking. Point-to-point selection is a particular case of point-to-group selection and can be treated as point-to-group selection with just one attempt to set up a connection. Hence, point-to-point selection leads to a decrease in the loss probability and, in particular, external blocking probability as compared to point-to-group selection.

\section{Models of output links of a switching network}

\subsection{Introduction}

Losses in the switching network are due to the phenomenon of internal and external blocking. To shape the dependence between the values of losses in individual multiservice traffic classes, it is possible to introduce threshold mechanisms [51] and resource reservation mechanisms [40] to particular links in switching networks. These mechanisms can be treated as both executions of the call admission control function and resource management processes, thus making it possible to introduce dynamic resource reservation for particular traffic classes or appropriate adaptation of the volume of allocated resources to match the occupancy state of the system.

The assumption in the article is that, to reduce the complexity of algorithms for call admission control process in the switching network [10], resource reservation mechanisms and threshold mechanisms will be introduced to just output directions (groups of output links). However, it should be noticed that, on account of the algorithm for setting up connections [the first stage of the algorithm involves the identification of the availability of output links (Sect. 3.2)], an introduction of resource management mechanisms in output directions of the switching network also has direct influence on the change in traffic characteristics of intra-stage groups.

In order to present the proposed universal method for modelling switching networks with Erlang, Engset and Pascal traffic by resource reservation and threshold mechanisms, Sect. 4.2 presents an analytical model of a group of output directions in a switching network, whereas Sect. 5 includes a presentation of a new analytical model of inter-stage links in the switching network.

\subsection{Model of output links with resource reservation}

Consider the model of an output direction (a group of output links) presented in Sect. 3.1, the so-called limited-availability group model with multiservice traffic sources [54] (Fig. 2) and introduced reservation mechanisms. Let us notice that the considered model accurately describes the output directionpresented in Sect. 3.1-that is composed of $v$ identical separated links, each having the capacity of $f$ AUs. The total capacity of the group (output direction of the network) $V_{\mathrm{L}}$ is $V_{\mathrm{L}}=v f \mathrm{AUs}$.

In the considered system (the output direction of the switching network), a resource reservation mechanism for a given number of traffic classes has been introduced. The reservation mechanism has been defined for the set of classes $\mathbb{R}$, that is a subset of the set of all traffic classes $\mathbb{M}$, by a determination of the so-called reservation threshold $R_{c}$, i.e. 
the maximum occupancy state for the group in which a call of class $c$ that belongs to set $\mathbb{R}$ can still be admitted for service. Additionally, parameter $S_{c}$ has been defined that defines the reservation space, i.e. the set of occupancy states in which a call of class $c$ cannot be admitted for service: $S_{c}=V_{\mathrm{L}}-R_{c}$. According to the adopted reservation mechanism, the reservation threshold is determined for the occupancy state of the whole of the group irrespective of the actual occupancy state of individual links in the group.

The system with limited availability and resource reservation under consideration admits for service calls of class $c$ - that belongs to set $\mathbb{R}$ - only when a call can be entirely serviced by the resources of one of the links and when the number of free AUs in the group is higher than or equal to the value of reservation space $S_{c}$. A call of class $c$ that does not belong to set $\mathbb{R}$ can be admitted for service when it can be entirely serviced by the resources of one of the links (resources demanded to service a single call cannot be allocated in a number of links, which is a characteristic feature of systems with limited availability [54]). A group with limited availability is then a good example of a system with state-dependent service process in which the state dependence results from the structure of a group and the introduced reservation mechanism.

It is proved in [55] that in order to model multiservice systems with state-dependent call admission process one can apply an approximation of the service process that occurs in these systems by one-dimensional Markov chain. The adopted approach allows the Kaufman-Roberts recurrence $[27,28]$, developed for systems with state-independent call admission process and Erlang call streams, to be generalised to a form that makes it possible to determine the occupancy distribution (state probability) in systems with state dependent call arrival process servicing Erlang, Engset and Pascal traffic streams [55]:

$$
\begin{aligned}
n\left[P_{n}\right]_{V_{\mathrm{L}}}= & \sum_{i=1}^{s_{I}} \sum_{c=1}^{c_{\mathrm{Er}, i}} A_{\mathrm{Er}, i, c} \sigma_{c}\left(n-t_{c}\right) t_{c}\left[P_{n-t_{c}}\right] V_{\mathrm{L}} \\
& +\sum_{j=1}^{s_{J}} \sum_{c=1}^{c_{\mathrm{En}, j}} A_{\mathrm{En}, j, c}\left(n-t_{c}\right) \sigma_{c}\left(n-t_{c}\right) t_{c}\left[P_{n-t_{c}}\right] V_{\mathrm{L}} \\
& +\sum_{k=1}^{s_{K}} \sum_{c=1}^{c_{\mathrm{Pa}, k}} A_{\mathrm{Pa}, k, c}\left(n-t_{c}\right) \sigma_{c}\left(n-t_{c}\right) t_{c}\left[P_{n-t_{c}}\right] V_{\mathrm{L}} .
\end{aligned}
$$

In Formula (6), $\left[P_{n}\right]_{V_{\mathrm{L}}}$ is the occupancy distribution in a system with capacity $V_{\mathrm{L}}$, while parameter $\sigma_{c}(n)$ defines the total dependence of the call admission process in the system on the current occupancy state of the system, resulting from both the particular structure of the group and from the introduced reservation mechanism.
In order to reflect the influence of a specific structure of a group on the process of determining the occupancy distribution with the recurrence (6), it is proposed in [56] to introduce conditional transition coefficients $\sigma_{c, \mathrm{~S}}(n)$. The value of the parameter $\sigma_{c, \mathrm{~S}}(n)$ does not depend on the call arrival process and can be determined as follows [56]:

$\sigma_{c, \mathrm{~S}}(n)=\frac{F\left(V_{\mathrm{L}}-n, v, f, 0\right)-F\left(V_{\mathrm{L}}-n, v, t_{c}-1,0\right)}{F\left(V_{\mathrm{L}}-n, v, f, 0\right)}$,

where $F(x, v, f, t)$ determines the number of possible arrangements of $x$ free AUs in $v$ links, each with the capacity equal to $f$ AUs, with accompanying assumption that each link has at least $t$ free AUs:

$$
\begin{aligned}
F(x, v, f, t)= & \sum_{r=0}^{\left\lfloor\frac{x-v t}{f-t+1}\right\rfloor}(-1)^{r}\left(\begin{array}{l}
v \\
r
\end{array}\right) \\
& \left(\begin{array}{c}
x-v(t-1)-1-r(f-t+1) \\
v-1
\end{array}\right) .
\end{aligned}
$$

Let us notice that in the case of the considered model of a limited-availability group, multiservice traffic sources and reservation, the operation of the reservation mechanism introduces additional dependence between the service stream in the system and the current state of the system. To determine this dependence, parameter $\sigma_{c, \mathrm{R}}(n)$ is introduced. This parameter can be determined in the following way:

$\sigma_{c, \mathbb{R}}(n)= \begin{cases}1 & \text { for } n \leqslant R_{c} \wedge c \in \mathbb{R}, \\ 0 & \text { for } n>R_{c} \wedge c \in \mathbb{R}, \\ 1 & \text { for } c \notin \mathbb{R}\end{cases}$

The reservation mechanism is introduced to the group irrespective of the group structure, which makes it possible to use a product description of the total transition coefficient in a limited-availability group:

$\sigma_{c}(n)=\sigma_{c, \mathrm{~S}}(n) \cdot \sigma_{c, \mathrm{R}}(n)$.

With the occupancy distribution calculated and obtained on the basis of Formula (6), we are in a position to determine the values of parameters $y_{\mathrm{En}, j, c}(n)$ and $y_{\mathrm{Pa}, k, c}(n)$ which define the average number of serviced calls of class $c$ generated by Engset sources from set $j$ and Pascal sources from set $k$, respectively, in the occupancy state of $n$ AUs [55]:

$$
\begin{aligned}
& y_{\mathrm{En}, j, c}(n) \\
& \quad= \begin{cases}A_{\mathrm{En}, j, c}\left(n-t_{c}\right) \sigma_{c}\left(n-t_{c}\right)\left[P_{n-t_{c}}\right]_{V_{\mathrm{L}}} /\left[P_{n}\right]_{V_{\mathrm{L}}} & \text { for } n \leqslant V_{\mathrm{L}}, \\
0, & \text { for } n>V_{\mathrm{L}},\end{cases}
\end{aligned}
$$




$$
\begin{aligned}
& y_{\mathrm{Pa}, k, c}(n) \\
& \quad= \begin{cases}A_{\mathrm{Pa}, k, c}\left(n-t_{c}\right) \sigma_{c}\left(n-t_{c}\right)\left[P_{n-t_{c}}\right]_{V_{\mathrm{L}}} /\left[P_{n}\right]_{V_{\mathrm{L}}} & \text { for } n \leqslant V_{\mathrm{L}}, \\
0, & \text { for } n>V_{\mathrm{L}} .\end{cases}
\end{aligned}
$$

The knowledge of distribution $\left[P_{n}\right]_{V_{\mathrm{L}}}$ is required for parameters $y_{\mathrm{En}, j, c}(n)$ and $y_{\mathrm{Pa}, k, c}(n)$ to be determined, and, in turn, to determine distribution $\left[P_{n}\right]_{V_{\mathrm{L}}}$ it is necessary to know the values of parameters $y_{\mathrm{En}, j, c}(n)$ and $y_{\mathrm{Pa}, k, c}(n)$, which enables the determination of traffic values $A_{\mathrm{En}, j, c}(n)$ of class $c$ offered by Engset sources from set $j$ in the occupancy state of $n$ AUs and traffic $A_{\mathrm{Pa}, k, c}(n)$ of class $c$ offered by Pascal sources from set $k$ in the occupancy state of $n$ AUs [Formula (3)]. Equations (3), (6), (11) and (12), thus form a series of confounded equations. To solve a given set of confounded equations, it is necessary to use the iterative methods developed in $[25,55]$. Having determined the occupancy distribution in the limited-availability group with a reservation mechanism, the blocking probability for calls of class $c$ that belong to set $\mathbb{M}=\{1,2, \ldots, m\}$ can be expressed as follows:

$E_{c}=\sum_{n=0}^{V_{\mathrm{L}}}\left[P_{n}\right]_{V_{\mathrm{L}}}\left[1-\sigma_{c}(n)\right]$.

\subsection{Model of an output group with threshold mechanisms}

Let us now consider a model of output links of a switching network to which a threshold mechanism has been introduced, i.e. a model of a limited-availability group with threshold mechanisms. The considered threshold mechanism makes the size of allocated resources (allocation units) for individual service classes dependent on the occupancy state of a given system. In a threshold system, an increase in the load of the system is followed by a decrease in the size of allocated resources to calls of a given service class. This can ultimately lead to a prolongation of data transmission time (for elastic services, where data need to be transmitted in their entirety) or to a decrease in the quality of transmitted multimedia data (for adaptive services, in which a prolongation of data transmission time is not possible). However, when the load of the system decreases, the number of AUs required by calls of individual classes increases, while the average service time can be decreased.

Let us consider a model of a limited-availability group with threshold mechanisms [55] with capacity $V_{\mathrm{L}}$ (Fig. 3), that services $m$ traffic classes belonging to set $\mathbb{M}=$ $\{1,2, \ldots, m\}$ (Sect. 3). For each class from set $\mathbb{M}$, a set of thresholds is defined. The set of introduced thresholds, related to class $c$, can be presented in the following form: $\left\{Q_{c, 1}, Q_{c, 2}, \ldots, Q_{c, q_{c}}\right\}$, where $\left\{Q_{c, 1} \leq Q_{c, 2} \leq \cdots \leq\right.$ $\left.Q_{c, q_{c}}\right\}$. In the system, a given threshold area $u$ of class $c$

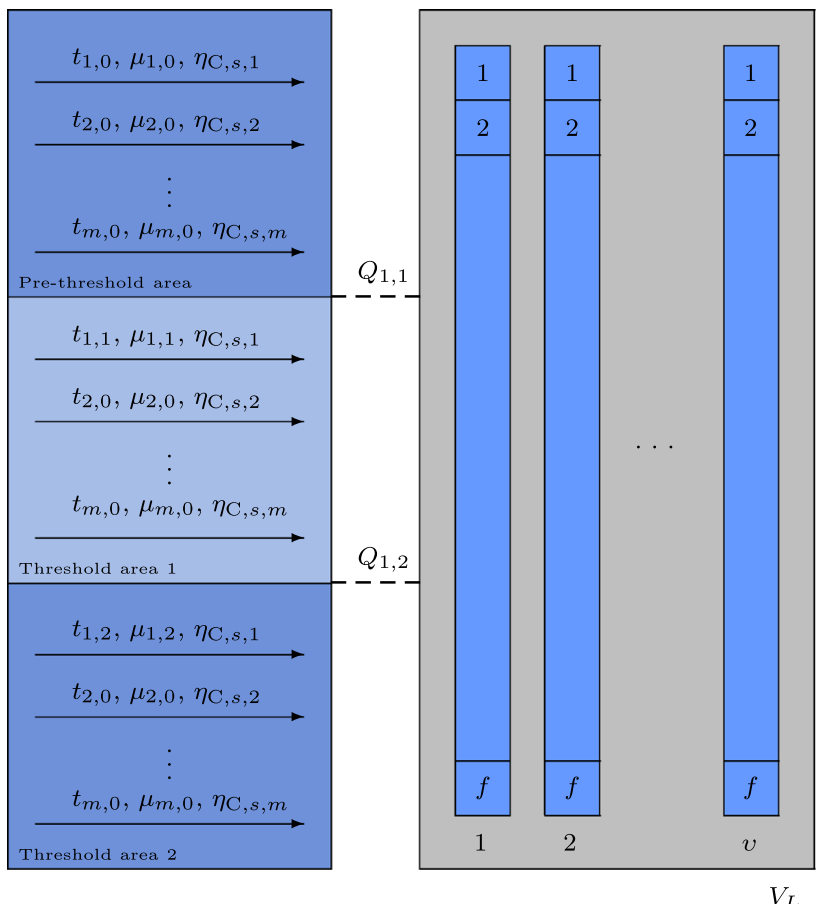

Fig. 3 Model of a limited-availability group with threshold mechanisms

limited by thresholds $Q_{c, u}$ and $Q_{c, u+1}$ is defined by its own set of parameters $\left\{t_{c, u}, \mu_{c, u}\right\}$, where $t_{c, 0}>t_{c, 1}>\cdots>$ $t_{c, u}>\cdots t_{c, q_{c}}$ and $\mu_{c, 0}^{-1} \leq \mu_{c, 1}^{-1} \leq \cdots \leq \mu_{c, u}^{-1} \leq \cdots \leq \mu_{c, q_{c}}$.

Figure 3 shows a sample model of a limited-availability group with threshold mechanisms in which a threshold mechanism has been introduced to class 1 only. In the pre-threshold area, calls of class $c$ demand $t_{c, 0}$ AUs to set up a connection, while the average service time is $\mu_{c, 0}^{-1}$. When the load of the system increases above the threshold value $Q_{c, 1}$, the system will be in the threshold area 1 . The number of demanded AUs will decrease from value $t_{c, 0}$ to value $t_{c, 1}$, while the average service time will increase to value $\mu_{c, 1}^{-1}$. The situation is similar when the system transfers to the next threshold areas.

The considered limited-availability group with threshold mechanisms is offered three types of traffic streams: Erlang, Engset and Pascal traffic streams, defined in Sect. 3.1. In order to include the influence of the threshold mechanisms on traffic value $A_{\mathrm{Er}, i, c, u}$, generated by Erlang sources that belong to set $\mathbb{Z}_{\mathrm{Er}, i}$, traffic value $A_{\mathrm{En}, j, c, u}(n)$ offered by Engset sources form set $\mathbb{Z}_{\mathrm{En}, j}$ and traffic $A_{\mathrm{Pa}, k, c, u}(n)$ offered by Pascal sources from set $\mathbb{Z}_{\mathrm{Pa}, k}$, in the threshold area $u$, Formulas (2) and (3) are to be adequately modified:

$$
\begin{aligned}
& A_{\mathrm{Er}, i, c, u}=\eta_{\mathrm{Er}, i, c} \lambda_{\mathrm{Er}, i} / \mu_{c, u}, \\
& A_{\mathrm{En}, j, c, u}(n)=\left[\eta_{\mathrm{En}, j, c} N_{\mathrm{En}, j}-y_{\mathrm{En}, j, c, u}(n)\right] \alpha_{\mathrm{En}, j, u}, \\
& A_{\mathrm{Pa}, k, c, u}(n)=\left[\eta_{\mathrm{Pa}, k, c} S_{\mathrm{Pa}, k}+y_{\mathrm{Pa}, k, c, u}(n)\right] \beta_{\mathrm{Pa}, k, u},
\end{aligned}
$$


where $\alpha_{\mathrm{En}, j, u}$ determines the average intensity of traffic offered by one free Engset source that belongs to set $\mathbb{Z}_{\mathrm{En}, j}$ in threshold area $u$ :

$\alpha_{\mathrm{En}, j, u}=\sum_{c=1}^{c_{\mathrm{En}, j}} \eta_{\mathrm{En}, j, c} \gamma_{\mathrm{En}, j} / \mu_{c, u}$,

whereas $\beta_{\mathrm{Pa}, k, u}$ determines the average traffic offered by one free Pascal source that belongs to set $\mathbb{Z}_{\mathrm{Pa}, k}$ in threshold area $u$ :

$\beta_{\mathrm{Pa}, k, u}=\sum_{c=1}^{c_{\mathrm{P}, k}} \eta_{\mathrm{Pa}, k, c} \gamma_{\mathrm{Pa}, k} / \mu_{c, u}$.

Observe that in the case of the considered model of limited-availability group with multiservice traffic sources and threshold mechanisms, the operation of the threshold mechanisms introduces an additional dependence between the service stream in the system and the current occupancy state of the system. To include this dependence in the considerations, in Eq. (6), we will introduce coefficient $\sigma_{c, u, \mathrm{P}}(n)$ that determines the occupancy states in the system in which offered traffic is defined by parameters $\left\{t_{c, u}, \mu_{c, u}\right\}$ :

$\sigma_{c, u, \mathrm{P}}(n)= \begin{cases}1 & \text { for } Q_{c, u}<n \leq Q_{c, u+1}, \\ 0 & \text { for remaining } n\end{cases}$

In addition, changing volumes of resources that are allocated to calls in particular occupancy states impose a change in the way the conditional transition coefficient $\sigma_{c, \mathrm{~S}}$ is determined [Formula (7)], i.e. the coefficient that describes the influence of the structure of the system on the new call admission process:

$\sigma_{c, \mathrm{~S}}(n)=\frac{F\left(V_{\mathrm{L}}-n, v, f, 0\right)-F\left(V_{\mathrm{L}}-n, v, t_{c, u}-1,0\right)}{F\left(V_{\mathrm{L}}-n, v, f, 0\right)}$,

where the value of function $F(x, v, f, t)$ is determined by Formula (8). In the case of Formula (19), value $t_{c, u}$ is matched according to the number of threshold $u$ determined on the basis of state $n$.

Observe that threshold mechanisms are introduced to the group regardless of its structure, which enables the product form description of the total transition coefficient in the limited-availability group:

$\sigma_{c, u}(n)=\sigma_{c, \mathrm{~S}}(n) \cdot \sigma_{c, u, \mathrm{P}}(n)$,

and then of the occupancy distribution in the considered threshold system:

$$
\begin{aligned}
n\left[P_{n}\right]_{V_{\mathrm{L}}}= & \sum_{i=1}^{s_{I}} \sum_{c=1}^{c_{\mathrm{Er}, i}} \sum_{u=1}^{q_{c}} A_{\mathrm{Er}, i, c, u} \sigma_{c, u}\left(n-t_{c, u}\right) t_{c, u}\left[P_{n-t_{c, u}}\right]_{V_{\mathrm{L}}} \\
+ & \sum_{j=1}^{s_{J}} \sum_{c=1}^{c_{\mathrm{En}, j}} \sum_{u=1}^{q_{c}} \\
& A_{\mathrm{En}, j, c, u}\left(n-t_{c, u}\right) \sigma_{c, u}\left(n-t_{c, u}\right) t_{c, u}\left[P_{n-t_{c, u}}\right]_{V_{\mathrm{L}}} \\
+ & \sum_{k=1}^{s_{K}} \sum_{c=1}^{c_{\mathrm{Pa}, k}} \sum_{u=1}^{q_{c}} \\
& A_{\mathrm{Pa}, k, c, u}\left(n-t_{c, u}\right) \sigma_{c, u}\left(n-t_{c, u}\right) t_{c, u}\left[P_{n-t_{c, u}}\right]_{V_{\mathrm{L}}} .
\end{aligned}
$$

Having calculated occupancies $\left[P_{n}\right]_{V_{\mathrm{L}}}$ from Formula (21), we are in the position to determine the values of the parameters $y_{\mathrm{En}, j, c, u}(n)$ and $y_{\mathrm{Pa}, k, c, u}(n)$ after applying the following equations:

$$
\begin{array}{r}
y_{\mathrm{En}, j, c, u}(n) \\
= \begin{cases}A_{\mathrm{En}, j, c, u}\left(n-t_{c, u}\right) \sigma_{c, u}\left(n-t_{c, u}\right)\left[P_{n-t_{c, u}}\right]_{V_{\mathrm{L}}} /\left[P_{n}\right]_{V_{\mathrm{L}}} & \text { for } n \leqslant V_{\mathrm{L}}, \\
0, & \text { for } n>V_{\mathrm{L}} ;\end{cases} \\
y_{\mathrm{Pa}, k, c, u}(n) \\
= \begin{cases}A_{\mathrm{Pa}, k, c, u}\left(n-t_{c, u}\right) \sigma_{c, u}\left(n-t_{c, u}\right)\left[P_{n-t_{c, u}}\right]_{V_{\mathrm{L}}} /\left[P_{n}\right]_{V_{\mathrm{L}}} & \text { for } n \leqslant V_{\mathrm{L}}, \\
0, & \text { for } n>V_{\mathrm{L}} .\end{cases}
\end{array}
$$

Just as in the case of the limited-availability group with reservation, Formulas (15), (21), (22) and (23) form a system of confounded equations. To solve them, the iterative process proposed in [57] has to be applied. Thus determined occupancy distribution allows us then to determine the blocking probability for each of the $m$ service classes. For calls of class $c$, this can be expressed by the following formula:

$E_{c}=\sum_{n=V_{\mathrm{L}}-t_{c, q_{c}}+1}^{V_{\mathrm{L}}}\left[P_{n}\right]_{V_{\mathrm{L}}}\left(1-\sigma_{c, \mathrm{~S}}(n)\right)$.

\section{Models of inter-stage links in a switching network}

\subsection{Introduction}

Let us now consider an analytical model of individual interstage links of the switching network. Given the adopted assumption, the algorithm that controls the call admission process admits of a new call (or the size of resources allocated to calls of particular classes) depending on the threshold mechanism that has been introduced to output directions only, while taking into account the current occupancy state 
of a given direction. During the second stage of the admission of a new call, i.e. while setting up a connection path between a free input link and one of the free output links in the demanded direction, the control device checks only if there is a connection path that offers a sufficient number of free AUs to service a call of class $c$ (the number of AUs can be decreased as a result of the application of threshold mechanisms in the output direction). It should be noticed though that the applied mechanisms that make the operation of the function of new call admission control dependent on the occupancy state of output directions has a direct influence on the structure of traffic offered to inter-stage links.

The assumption in most works devoted to analytical modelling of switching networks (e.g. [48]) is that inter-stage links can be described by the model of the so-called fullavailability group with multiservice traffic. The model of a full-availability group, without threshold mechanisms and/or reservation, was initially developed for Erlang traffic streams $[27,28]$, and then was expanded to include systems that serviced Engset and Pascal traffic streams [25]. Głąbowski and Sobieraj [51] assumes that in the case of the introduction of threshold mechanisms to output directions of a network, inter-stage links can be modelled by the model of a fullavailability group with threshold mechanisms. Within this approach, it is necessary to determine the values of thresholds $Q_{c, u}^{\mathrm{F}}$ for the full-availability group that influence the size of resources allocated to calls of class $c$, depending on the current occupancy distribution. The basic problem in determining these values results from the difference in capacity $V_{\mathrm{L}}$ of the output direction and $V_{\mathrm{F}}$ of a single inter-stage link $\left(V_{\mathrm{F}}=f, V_{\mathrm{L}}=v f\right)$. To reflect the values of the thresholds established for the model of a limited-availability group (model of a group of output links) on the values of the thresholds implemented in the model of a full-availability group (model of a single link), in [51] the following approximation is proposed:

$Q_{c, u}^{\mathrm{F}}=\left\lfloor Q_{c, u}^{\mathrm{L}} / v\right\rfloor$,

where $Q_{c, u}^{\mathrm{F}}$ is the threshold value in the output direction, while $Q_{c, u}^{\mathrm{F}}$ is a fictitious threshold value in an inter-stage link.

\subsection{A new model of inter-stage links with threshold mechanisms}

This section presents a new method of modelling inter-stage links in switching networks where threshold mechanisms and reservation mechanisms have been introduced to the output directions. The assumption in the adopted method is that inter-stage links will be modelled by the model of a fullavailability group without threshold mechanisms [46]. The influence of the threshold mechanisms introduced in output links on the structure of traffic in inter-stage links will be taken into account by a change in the number of traffic classes offered to inter-stage links. In these circumstances, calls of class $c$ that are offered to output links can have $\left(q_{c}+1\right)$ different $t_{c, u}$ values of resources (AUs) allocated, depending on the occupancy state of the system, where $u$ is the threshold number and $q_{c}$ is the number of thresholds defined for class $c$. A given number of resource demands that can occur in output directions as a result of the applied threshold mechanisms determines at the same time the maximum number of traffic classes whose calls can be offered to inter-stage links. Observe that the number of defined sets of $s_{I}$ Erlang, $s_{J}$ Engset and $s_{K}$ Pascal traffic sources does not change, but it is only the number of traffic classes that belong respectively to appropriate sets $\mathbb{C}_{\mathrm{Er}, i}, \mathbb{C}_{\mathrm{En}, j}$ and $\mathbb{C}_{\mathrm{Pa}, k}$ of traffic classes that correspond to appropriate sets of multiservice traffic sources, that can be changed. An increased count of sets $\mathbb{C}_{\mathrm{Er}, i}, \mathbb{C}_{\mathrm{En}, j}$ and $\mathbb{C}_{\mathrm{Pa}, k}$ can be expressed by the following equations:

$c_{\mathrm{Er}, i}^{\mathrm{F}}=\sum_{c=1}^{c_{\mathrm{Er}, i}}\left(q_{c}+1\right)$,

$c_{\mathrm{En}, j}^{\mathrm{F}}=\sum_{c=1}^{c_{\mathrm{En}, j}}\left(q_{c}+1\right)$,

$c_{\mathrm{Pa}, k}^{\mathrm{F}}=\sum_{c=1}^{c_{\mathrm{Pa}, k}}\left(q_{c}+1\right)$.

Parameters $t_{c}$ and $\mu_{c}$ for new traffic classes related to call streams offered to the full-availability group can be obtained by establishing a particular relationship between parameters $t_{c, u}$ and $\mu_{c, u}$ that are related to call streams offered to the limited-availability group in individual threshold areas. This particular assignment of appropriate values to parameters $t_{c}$ and $\mu_{c}$ is performed on the basis of the following formulas:

$\forall_{1 \leq c \leq m} \quad \forall_{0 \leq u \leq q_{c}} t_{c+u+\sum_{z=1}^{c-1} q_{z}}=t_{c, u}$,

$\forall_{1 \leq c \leq m} \forall_{0 \leq u \leq q_{c}} \mu_{c+u+\sum_{z=1}^{c-1} q_{z}}=\mu_{c, u}$.

In Formulas (27) and (28), expression $\sum_{z=1}^{c-1} q_{z}$ prevents us from making unnecessary duplicate indexes related to the new traffic classes. The applied method for determining the value of parameters $t_{c}$ and $\mu_{c}$ makes it possible to use a full-availability group without threshold mechanisms to approximate inter-stage links of switching networks with threshold mechanisms.

Let us now consider a method of determining the intensity of traffic offered to a new class $c$ in an inter-stage link. For this purpose, let us introduce parameter $b_{c, u}$ to the model under consideration which determines the blocking probability for calls of class $c$ to which a given number of AUs, related to 


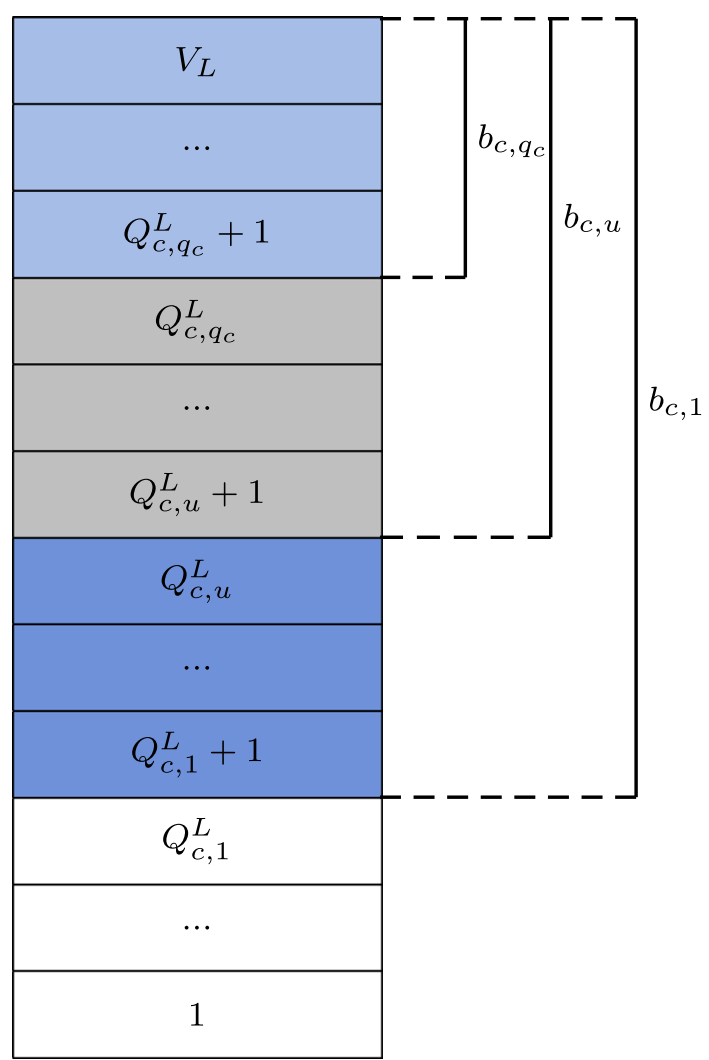

Fig. 4 Determination of parameter $b_{c, u}$ in limited-availability group

threshold area $u-1$, is allocated. The values of parameter $b_{c, u}$ can be determined on the basis of the following formula:

$b_{c, u}=\sum_{n=Q_{c, u}^{\mathrm{L}}+1}^{V_{\mathrm{L}}}\left[P_{n}\right]_{V_{\mathrm{L}}}$.

Parameter $b_{c, u}$ is equal to the sum of the probabilities of these states (Fig. 4) in which it is not feasible to allocate resources equal to: $\left(t_{c, u-1}, t_{c, u-2}, \ldots, t_{c, 0}\right)$ to a call of class $c$. Occupancy distribution $\left[P_{n}\right]_{V_{\mathrm{L}}}$ is a distribution determined on the basis of the model of a limited-availability group and threshold mechanisms (Sect. 4.3). Let us assume that an output group of the switching network is offered traffic $A_{\mathrm{Er}, i, c, 0}$ of class $c$ that demands $t_{c, 0}$ AUs. This means that in the interstage link we will define "new traffic" of class $c$, demanding $t_{c+0}=t_{c, 0}$ AUs to set up a connection. This traffic is part of traffic $A_{\mathrm{Er}, i, c, 0}$ and can be evaluated on the basis of the following reasoning: in the pre-threshold area of the output group, this part of traffic $A_{\mathrm{Er}, i, c, 0}$ that demands $t_{c, 0} \mathrm{AUs}$ is serviced that is not blocked in this area: $A_{\mathrm{Er}, i, c, 0}\left(1-b_{c, 1}\right)$. The first threshold area of the output group is offered traffic that is blocked in the pre-threshold area: $A_{\mathrm{Er}, i, c, 0} b_{c, 1}$. This results in the arrival of demands with the value $t_{c+1}=t_{c, 1}$ in the inter-stage link. Consequently, traffic that is blocked in the threshold areas, from zero threshold area (in the prethreshold area) to $u-1$, i.e. traffic $A_{\mathrm{Er}, i, c, 0} \prod_{q=1}^{u} b_{c, q}$, is directed to threshold area $u$. Part of this particular traffic that is not blocked in area $u$ will create "new" traffic of class $c$ with the value:

$$
\left(1-b_{c, u+1}\right) A_{\mathrm{Er}, i, c, 0} \prod_{q=1}^{u} b_{c, q}
$$

Observe that traffic described by Formula (30), demanding $t_{c, u} \mathrm{AUs}$, is determined in the output group of the switching network that is composed of $v$ links. Therefore, traffic over one link that demands $t_{c+u}$ AUs will be $v$ times lower:

$\frac{1}{v}\left(1-b_{c, u+1}\right) A_{\mathrm{Er}, i, c, 0} \prod_{q=1}^{u} b_{c, q}$.

By taking the above reasoning into consideration and taking into account the total traffic offered to the inter-stage link, we can write:

$$
\begin{aligned}
\forall_{1 \leq i} \leq s_{I} & \forall_{1 \leq c \leq c_{\mathrm{Er}, i}} \forall_{0 \leq u \leq q_{c}} A_{\mathrm{Er}, i, c+u+\sum_{z=1}^{c-1} q_{z}}^{\mathrm{F}} \\
= & \left\{\begin{array}{lll}
{\left[A_{\mathrm{Er}, i, c, 0}\left(1-b_{c, u+1}\right) \prod_{z=1}^{u} b_{c, z}\right] / v} & \text { for } u<q_{c}, \\
{\left[A_{\mathrm{Er}, i, c, 0} \prod_{z=1}^{u} b_{c, z}\right] / v} & \text { for } u=q_{c},
\end{array}\right.
\end{aligned}
$$

where the average traffic intensity $A_{\mathrm{Er}, i, c, 0}$ is determined on the basis of Formula (14), whereas that of the parameter $b_{c, u}$ is on the basis of Formula (29).

Following the same reasoning, the average traffic $\alpha_{\mathrm{En}, j, c}^{\mathrm{F}}$ and $\beta_{\mathrm{Pa}, k, c}^{\mathrm{F}}$ offered by one free source from a set of traffic sources of Engset and Pascal traffic, respectively, can be determined:

$$
\begin{aligned}
& \forall_{1 \leq j \leq s_{J}} \forall_{1 \leq c \leq c_{\mathrm{En}, j}} \forall_{0 \leq u \leq q_{c}} \alpha_{\mathrm{En}, j, c+u+\sum_{z=1}^{c-1} q_{z}}^{\mathrm{F}} \\
& = \begin{cases}{\left[\alpha_{\operatorname{En}, j, c, 0}\left(1-b_{c, u+1}\right) \prod_{z=1}^{u} b_{c, z}\right] / v} & \text { for } u<q_{c}, \\
{\left[\alpha_{\operatorname{En}, j, c, 0} \prod_{z=1}^{u} b_{c, z}\right] / v} & \text { for } u=q_{c},\end{cases} \\
& \forall_{1 \leq k \leq s_{K}} \forall_{1 \leq c \leq c \mathrm{~Pa}, k} \forall_{0 \leq u \leq q_{c}} \beta_{\mathrm{Pa}, k, c+u+\sum_{z=1}^{c-1} q_{z}}^{\mathrm{F}} \\
& = \begin{cases}{\left[\beta_{\mathrm{Pa}, k, c, 0}\left(1-b_{c, u+1}\right) \prod_{z=1}^{u} b_{c, z}\right] / v} & \text { for } u<q_{c}, \\
{\left[\beta_{\mathrm{Pa}, k, c, 0} \prod_{z=1}^{u} b_{c, z}\right] / v} & \text { for } u=q_{c},\end{cases}
\end{aligned}
$$

where the average intensity of traffic $\alpha_{\mathrm{En}, j, c, 0}$ and $\beta_{\mathrm{Pa}, k, c, 0}$ offered by one free Engset and Pascal source in the prethreshold area is determined on the basis of the following formulas:

$\alpha_{\mathrm{En}, j, c, 0}=\eta_{\mathrm{En}, j, c} \gamma_{\mathrm{En}, j} / \mu_{c, 0}$,

$\beta_{\mathrm{Pa}, k, c, 0}=\eta_{\mathrm{Pa}, k, c} \gamma_{\mathrm{Pa}, k} / \mu_{c, 0}$. 
Having determined the values for traffic: $A_{\mathrm{Er}, i, c}^{\mathrm{F}}, \alpha_{\mathrm{En}, j, c}^{\mathrm{F}}$ and $\beta_{\mathrm{Pa}, k, c}^{\mathrm{F}}$, it is possible to determine occupancy distribution $\left[P_{n}\right]_{V_{\mathrm{F}}}$ in the inter-stage link:

$$
\begin{aligned}
n\left[P_{n}\right]_{V_{\mathrm{F}}}= & \sum_{i=1}^{s_{I}} \sum_{c=1}^{c_{\mathrm{Er}, i}^{\mathrm{F}}} A_{\mathrm{Er}, i, c}^{\mathrm{F}} t_{c}\left[P_{n-t_{c}}\right]_{V_{\mathrm{F}}} \\
& +\sum_{j=1}^{s_{J}} \sum_{c=1}^{c_{\mathrm{En}, j}^{\mathrm{F}}} A_{\mathrm{En}, j, c}^{\mathrm{F}}\left(n-t_{c}\right) t_{c}\left[P_{n-t_{c}}\right]_{V_{\mathrm{F}}} \\
& +\sum_{k=1}^{s_{K}} \sum_{c=1}^{c_{\mathrm{Pa}, k}^{\mathrm{F}}} A_{\mathrm{Pa}, k, c}^{\mathrm{F}}\left(n-t_{c}\right) t_{c}\left[P_{n-t_{c}}\right]_{V_{\mathrm{F}}} .
\end{aligned}
$$

In Formula (37), parameter $A_{\mathrm{En}, j, c}^{\mathrm{F}}(n)$ determines the average intensity of offered traffic, in state $n$ busy AUs, by calls of class $c$, generated by the sources from the Engset set:

$A_{\mathrm{En}, j, c}^{\mathrm{F}}(n)=\left[\eta_{\mathrm{En}, j, c} N_{\mathrm{En}, j}-y_{\mathrm{En}, j, c}^{\mathrm{F}}(n)\right] \alpha_{\mathrm{En}, j}^{\mathrm{F}}$,

where parameter $\alpha_{\mathrm{En}, j}^{\mathrm{F}}$ is determined by the following formula:

$\alpha_{\mathrm{En}, j}^{\mathrm{F}}=\sum_{c=1}^{c_{\mathrm{En}, j}^{\mathrm{F}}} \alpha_{\mathrm{En}, j, c}^{\mathrm{F}}$,

in which parameter $\alpha_{\mathrm{En}, j, c}^{\mathrm{F}}$ is determined on the basis of Formula (33). Parameter $A_{\mathrm{Pa}, k, c}^{\mathrm{F}}(n)$ in Formula (37) is then the average intensity of traffic offered in state $n$ by calls of class $c$ generated by sources from the Pascal set:

$A_{\mathrm{Pa}, k, c}^{\mathrm{F}}(n)=\left[\eta_{\mathrm{Pa}, k, c} S_{\mathrm{Pa}, k}+y_{\mathrm{Pa}, k, c}^{\mathrm{F}}(n)\right] \beta_{\mathrm{Pa}, k}^{\mathrm{F}}$,

where parameter $\beta_{\mathrm{Pa}, k}^{\mathrm{F}}$ is determined by the following equation:

$\beta_{\mathrm{Pa}, k}^{\mathrm{F}}=\sum_{c=1}^{c_{\mathrm{Pa}, k}^{\mathrm{F}}} \beta_{\mathrm{Pa}, k, c}^{\mathrm{F}}$,

in which parameter $\beta_{\mathrm{Pa}, k, c}^{\mathrm{F}}$ is determined by Formula (34).

With the occupancy distribution calculated and obtained on the basis of Formula (37), it is possible to determine the values of parameters $y_{\mathrm{En}, j, c}^{\mathrm{F}}(n)$ and $y_{\mathrm{Pa}, k, c}^{\mathrm{F}}(n)$ that indicate the average number of calls of class $c$ that are serviced in the system, generated by the sources that belong to sets $\mathbb{Z}_{\mathrm{En}, j}$ (Engset source) and $\mathbb{Z}_{\mathrm{Pa}, k}$ (Pascal source):

$$
\begin{aligned}
& y_{\mathrm{En}, j, c}^{\mathrm{F}}(n) \\
& \quad=\left\{\begin{array}{lll}
A_{\mathrm{En}, j, c}^{\mathrm{F}}\left(n-t_{c}\right)\left[P_{n-t_{c}}\right]_{V_{\mathrm{F}}} /\left[P_{n}\right]_{V_{\mathrm{F}}} & \text { for } & n \leqslant V_{\mathrm{F}}, \\
0 & \text { for } & n>V_{\mathrm{F}},
\end{array}\right.
\end{aligned}
$$

$$
\begin{aligned}
& y_{\mathrm{Pa}, k, c}^{\mathrm{F}}(n) \\
& \quad=\left\{\begin{array}{lll}
A_{\mathrm{Pa}, k, c}^{\mathrm{F}}\left(n-t_{c}\right)\left[P_{n-t_{k}}\right]_{V_{\mathrm{F}}} /\left[P_{n}\right]_{V_{\mathrm{F}}} & \text { for } & n \leqslant V_{\mathrm{F}}, \\
0 & \text { for } & n>V_{\mathrm{F}} .
\end{array}\right.
\end{aligned}
$$

To determine parameters $y_{\mathrm{En}, j, c}^{\mathrm{F}}(n)$ and $y_{\mathrm{Pa}, k, c}^{\mathrm{F}}(n)$ it is necessary to know occupancy distribution $\left[P_{n}\right]_{V_{\mathrm{F}}}$; finding it, in turn, requires the values of parameters $y_{\mathrm{En}, j, c}^{\mathrm{F}}(n)$ and $y_{\mathrm{Pa}, k, c}^{\mathrm{F}}(n)$ to be known. Therefore, to determine occupancy distribution $\left[P_{n}\right]_{V_{\mathrm{F}}}$, the iterative method, developed in [25], has to be applied. In this method, traffic values in a given step of iteration $A_{\mathrm{En}, j, c}^{\mathrm{F}}(n)$ and $A_{\mathrm{Pa}, k, c}^{\mathrm{F}}(n)$ are determined on the basis of parameters $y_{\mathrm{En}, j, c}^{\mathrm{F}}(n) \mathrm{i} y_{\mathrm{Pa}, k, c}^{\mathrm{F}}(n)$ determined in the preceding iteration step.

Blocking probability in the inter-stage link is equal to the sum of probabilities of states in which the system cannot admit new calls of class $c$ for service. Therefore, blocking probability can be expressed by the following formula:

$E_{c}^{\mathrm{F}}=\sum_{n=V_{\mathrm{F}}-t_{c}+1}^{V_{\mathrm{F}}}\left[P_{n}\right]_{V_{\mathrm{F}}}$,

where occupancy distribution $\left[P_{n}\right]_{V_{\mathrm{F}}}$ is determined by Formula (37).

\section{Recurrent method for the determination of blocking probability in a switching network}

Total blocking probability $E_{\mathrm{T}}(c)$ for calls of class $c$ is the sum of all external blocking probabilities $E_{\mathrm{E}}(c)$ and internal blocking probability $E_{\mathrm{I}}(c)$. By taking into consideration the dependencies between the events of internal and external blocking, we get [58]:

$E_{\mathrm{T}}(c)=E_{\mathrm{E}}(c)+E_{\mathrm{I}}(c)\left[1-E_{\mathrm{E}}(c)\right]$.

The difference $\left[1-E_{\mathrm{E}}(c)\right]$ in the second element of Formula (45) results from the operation of a control device that controls a switching network. This device first checks if there are any free output links in a given direction for calls of class $c$. If such links cannot be found, then the call is lost due to external blocking and there is no need to check whether internal blocking occurs or not. Such an algorithm is determined by Formula (45) in which a concurrence of events of external and internal blocking is excluded.

External blocking occurs when none of the output links in a demanded direction of a switching network is not capable of serving a call of class $c$. Consequently, external blocking probability is equal to the blocking probability in the limited-availability group that models the output direction 
of the switching network and can be determined, depending on the admission control mechanism and resource allocation mechanisms applied, on the basis of Eq. (13) (for reservation) or (24) (for threshold mechanisms).

In order to determine the internal blocking probability, the article proposes a recurrent method-the PGPPBRecU method (Point-to-Group and Point-to-Point Blocking Recurrent-Universal) that takes into consideration a generalised model of inter-stage links in switching networks with multiservice traffic sources and reservation and threshold mechanisms. This method is a modification of the recurrent method described in [48] and developed to determine pointto-group and point-to-point blocking probability in switching networks with single-service traffic sources.

The proposed method is based on the effective availability parameter. This parameter averages out the average number of switches of the last stage with which a call in a given direction can be set up. The assumption is that the switch of the last stage can be directly available or available through direct occupancy. In line with definition [32], a switch is directly available when, in a given state of the network, there is a possibility of setting up a connection of a given class between this switch and a given switch of the first stage (at the input of which a call arrives). A switch is available through direct occupancy if there is a set up connection of a given class between this switch and a given switch of the first stage.

The determination of the effective availability for a traffic stream of class $c$ is based on the concept of the so-called equivalent network [32]. The equivalent network is a singleservice network with the same structure as the multiservice network. Each single link of the equivalent network is assigned a fictitious load $e_{l}(c)$ that is equal to the blocking probability for traffic of class $c$ in an inter-stage link of a real network, between stages $l$ and $l+1$. This probability can be determined on the basis of occupancy distribution in the full-availability group that models inter-stage links of the switching network [Formula (44), Sect. 5]. For the purpose of the discussed recurrent method, to determine effective availability $d_{z}(c)$ for a traffic stream of class $c$ in $z$-stage equivalent network, the following formula can be used [48]:

$d_{z}(c)=\left[1-\pi_{z}(c)\right] v+\pi_{z}(c) e_{1}(c)$.

where

$-d_{z}(c)$ - effective availability for traffic stream of class $c$ in equivalent $z$-stage switching network,

- $\pi_{z}(c)$ - the so-called probability of direct unavailability of a given switch of the last stage for a call of class $c$. Parameter $\pi_{z}(c)$ determines the probability of an event in which a connection of class $c$ between a given switch of the first stage and a given switch of the last stage cannot be set up. The determination of this parameter is based on the analysis of a channel graph for the equivalent switching network [32,59],

$-v$-the number of output links in a given direction,

- $\eta$ - the value that defines which part of fictitious traffic, in a switch of the first stage, is serviced by the considered direction. If traffic is uniformly distributed between all $v$ directions, we get $\eta=1 / v$.

In Formula (46), the first element $\left[1-\pi_{z}(c)\right] v$ determines the average number of switches of the last stage that are directly available to a switch of the first stage, whereas the second element $\pi_{z}(c) e_{1}(c)$ defines the average number of switches of the last stage available through direct occupancy [60].

To present the basic assumptions for this method, let us consider the $z$-stage switching network presented in Fig. 5. It is observable, having in mind Lotze's theorem [61], that unavailability probability $\pi_{l}(c)$ for a switch of stage $l$ for calls of class $c$ is equal to the blocking probability in the group of $v$ input links in the switch, i.e. to the point-to-group blocking probability $E_{l-1}$ in a switching network that is composed of $(l-1)$ stages:

$\pi_{l}(c)=E_{l-1}(c)$.

The effective availability parameter $d_{l}(c)$ for calls of class $c$ in a sub-system consisting of $l$ stages of a $z$-stage switching network is a function dependable on unavailability probability $\pi_{l}(c)$ of a switch of stage $l$ to calls of class $c$, and in this way [Eq. (47)] it is a function dependent on the pointto-group blocking probability $E_{l-1}$ in a sub-system that is composed of $(l-1)$ stages.

The assumption in the PGPPBRec-U method is that the point-to-group internal blocking probability $E_{\mathrm{I}}$ in an equivalent switching network can be approximated by the EIF formula (Erlang's Interconnection Formula) [36] proposed by Ershov to model switching networks with single-service traffic [36]:

$E_{\mathrm{I}}=\operatorname{EIF}(A, v, d)$,

where $A$ is offered traffic in the demanded direction of the equivalent switching network, $v$ is the number of links in the demanded directions of the equivalent switching network, while EIF formula determines the blocking probability in Erlang's Ideal Grading with capacity $v$, availability $d$ and offered traffic $A$ :

$$
\begin{aligned}
& \operatorname{EIF}(A, v, d) \\
& \quad=\sum_{l=d}^{v} \frac{\left(\begin{array}{l}
l \\
d
\end{array}\right)}{\left(\begin{array}{l}
v \\
d
\end{array}\right)} \frac{A^{l}}{l !} \prod_{k=d}^{l-1}\left[1-\frac{\left(\begin{array}{l}
k \\
d
\end{array}\right)}{\left(\begin{array}{l}
v \\
d
\end{array}\right)}\right] / \sum_{j=0}^{v} \frac{A^{j}}{j !} \prod_{k=d}^{j-1}\left[1-\frac{\left(\begin{array}{l}
k \\
d
\end{array}\right)}{\left(\begin{array}{l}
v \\
d
\end{array}\right)}\right] .
\end{aligned}
$$


Fig. 5 Model of a $z$-stage switching network
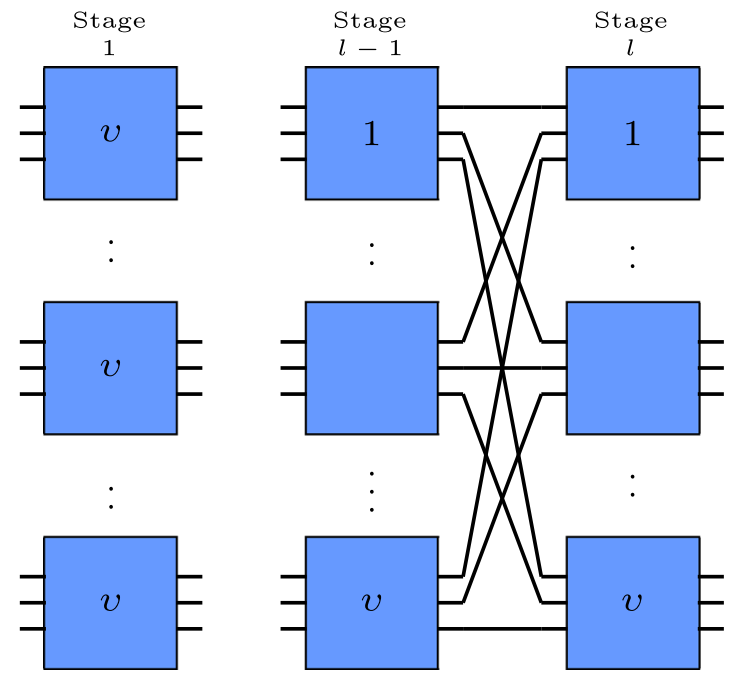

While considering the model of a switching network presented in Fig. 5, we can rewrite Eq. (48) into a form that would allow us to determine point-group internal blocking probabilities for calls of class $c$ in an equivalent switching network that is composed of $l$ stages:

$E_{\mathrm{I}, l}(c)=\operatorname{EIF}\left(A_{l+1}(c), v, d_{l}(c)\right)$,

where $A_{l+1}(c)$ is the traffic offered to one switch of stage $l+1$ by calls of class $c$, whereas $v$ is the number of input links to one switch of stage $l+1$.

Equations (47) and (50) provide the basis for recurrent determination of internal blocking probability in a multiservice switching network. The computational process [48] starts with the determination of blocking probability $E_{1}(c)$. Assuming a non-blocking nature of a switch of the first stage, we get that $d_{1}(c)=v$ and, in consequence:

$E_{\mathrm{I}, 1}(c)=\operatorname{EIF}\left(A_{2}(c), v, v\right)$,

where $A_{2}(c)$ determines the traffic offered to one switch of the second stage of the switching network by calls of class $c$ :

$A_{2}(c)=v a(c)$,

whereas $a(c)$ is the traffic of class $c$ offered to one interstage link in the switching network with the capacity of 1 AU. Offered traffic $a(c)$ can be determined on the basis of fictitious load $e(c)$ :

$e(c)=a(c)\left[1-E_{1}(a(c))\right]$,

where

- $E_{1}(a(c))$ is the blocking probability in inter-stage links of the switching network (between stages 1 and 2), deter- mined for a group with the capacity of $1 \mathrm{BBU}$ to which traffic $a(c)$ is offered,

$-e(c)$ is a fictitious load for calls of class $c$ in inter-stage links (between stages 1 and 2). This load is equal to the blocking probability for calls of class $c$ in an inter-stage link of the real network, modelled by a full-availability group with multiservice traffic sources (Sect. 5).

According to the proposed computational method, internal blocking probability $E_{\mathrm{I}, z}(c)$ in a $z$-stage network can be expressed as follows:

$E_{\mathrm{I}, z}(c)=E_{\mathrm{I}}(c)=\operatorname{EIF}\left(A(c), v, d_{z}(c)\right)$,

where $A(c)=A_{z+1}(c)$ is the total traffic offered by calls of class $c$ in the demanded direction, composed of $v$ links (according to the adopted notation, traffic offered to a given switch can be treated as traffic to one switch of a fictitious stage $z+1)$.

Observe that in order to evaluate the value of parameter $\pi_{l}(c)$ it is necessary to determine the blocking probability of the $(l-1)$ stage system [Formula (47)]. A solution to this problem can be provided by applying a recurrent algorithm.

The PGPPBRec-U recurrent method makes it possible to determine the blocking probability in switching networks with point-to-group selection and multiservice traffic sources. In addition, taking into consideration Lotze's assumptions [61] that the point-to-point blocking probability in a $z$-stage switching network is numerically equal to the point-to-group blocking probability in a $(z-1)$-stage network, the PGPPBRec-U method can provide a basis for a determination of the blocking probability in networks with the point-to-point selection. On the basis of the above considerations, the PGPPBRec-U method of determining point-to-group and point-to-point blocking probability by a 
generalised model of the switching network with threshold mechanisms can be written in the following form:

\section{PGPPBRec-U method}

1. Determination of external blocking probability on the basis of a model of limited-availability group with threshold mechanisms or reservation mechanisms-Formulas (24) or (13)

2. Initialization of iteration step $l=1$

3. Calculation of fictitious load in inter-stage links of switching networks on the basis of a generalised model of inter-stage links-Formula (44)

4. Calculation of the value of offered traffic-Formula (52)

5. Calculation of blocking probability in a single switch-Formula (48)

6. Increase of iteration step: $l=l+1$

7. Determination of effective availability parameter $d_{l}(c)$ in $l$-stage switching network with multiservice traffic sourcesFormula (46)

8. Determination of internal blocking probability in the switching network for individual call classes-Formula (54)

9. Repetition of steps 7-9:

point-to-group internal blocking, iterative process terminates when $l=z$,

point-to-point internal blocking, iterative process terminates when $l=z-1$

10. Calculation of total blocking probability-Formula (45)

\section{Numerical results}

The proposed method of determining the traffic characteristics of the considered switching networks is an approximate method. Hence, in order to evaluate the accuracy of the proposed method, the results of analytical modelling were compared with the data of simulation experiments and with the results obtained on the basis of analytical methods developed earlier. The simulations were carried out for a typical three-stage Clos switching network (Fig. 1) considered both in electronic and optical switching. The considered switching network was composed of switches with $v \times v$ links.

The results of the simulation are shown in the charts with $95 \%$ confidence intervals calculated according to the $t$-Student distribution for five series with $1,000,000$ calls of each class. For each of the points of the simulation, the value of the confidence interval is at least one order lower than the mean value of the results of the simulation. In a large number of instances, the value of the confidence interval is lower than the height of the sign used to indicate the value of the simulation experiment.

The research was carried out for four different structures of switching networks servicing three or four traffic classes:

\section{- System 1-Threshold mechanism:}

- structure of switching network: $v=4, f=42$ AUs, $V=168$ AUs;
- structure of offered traffic:

- traffic classes: $m=4, t_{1,0}=1 \mathrm{AU}, \mu_{1,0}^{-1}=1$, $t_{2,0}=6$ AUs, $\mu_{2,0}^{-1}=1, t_{3,0}=10 \mathrm{AUs}, \mu_{3,0}^{-1}=$ $1, t_{3,1}=8$ AUs, $\mu_{3,0}^{-1}=1,25, t_{4,0}=12$ AUs, $\mu_{4,0}^{-1}=1, t_{4,1}=8$ AUs, $\mu_{4,1}^{-1}=1,5$;

- sets of traffic sources: $S=2, \mathbb{C}_{\mathrm{Pa}, 1}=\{1,2,3\}$, $\eta_{\mathrm{Pa}, 1,1}=0,6, \eta_{\mathrm{Pa}, 1,2}=0,2, \eta_{\mathrm{Pa}, 1,3}=0,2$, $S_{\mathrm{Pa}, 1}=950, \mathbb{C}_{\mathrm{En}, 2}=\{1,2,4\}, \eta_{\mathrm{En}, 2,1}=0,6$, $\eta_{\mathrm{En}, 2,2}=0,2, \eta_{\mathrm{En}, 2,4}=0,2, N_{\mathrm{En}, 2}=950$;

- threshold mechanism: $Q_{3,1}=Q_{4,1}=126$ AUs.

- System 2-Threshold mechanism:

- structure of switching network: $v=4, f=28$ AUs, $V=112$ AUs;

- structure of offered traffic:

- traffic classes: $m=4, t_{1,0}=1 \mathrm{AU}, \mu_{1,0}^{-1}=1$, $t_{2,0}=2$ AUs, $\mu_{2,0}^{-1}=1, t_{3,0}=4$ AUs, $\mu_{3,0}^{-1}=1$, $t_{4,0}=8$ AUs, $\mu_{4,0}^{-1}=1, t_{4,1}=4$ AUs, $\mu_{4,1}^{-1}=2$, $t_{5,0}=12$ AUs, $\mu_{5,0}^{-1}=1, t_{5,1}=8$ AUs, $\mu_{5,1}^{-1}=$ $1,5, t_{5,2}=4$ AUs, $\mu_{5,2}^{-1}=3$;

- sets of traffic sources: $S=3, \mathbb{C}_{\mathrm{Er}, 1}=\{1,3\}$, $\eta_{\mathrm{Er}, 1,1}=0,6, \eta_{\mathrm{Er}, 1,3}=0,4, \mathbb{C}_{\mathrm{En}, 2}=\{1,2,4\}$, $\eta_{\mathrm{En}, 2,1}=0,5, \eta_{\mathrm{En}, 2,2}=0,3, \eta_{\mathrm{En}, 2,4}=0,2$, $N_{\text {En }, 2}=1000, \mathbb{C}_{\text {En }, 3}=\{1,4,5\}, \eta_{\text {En }, 3,1}=0,6$, $\eta_{\mathrm{En}, 3,4}=0,1, \eta_{\mathrm{En}, 3,5}=0,3, N_{\mathrm{En}, 3}=1000$;

- threshold mechanism: $Q_{4,1}=Q_{5,1}=84$ AUs, $Q_{5,2}=72$ AUs.

\section{- System 3-Bandwidth reservation mechanism:}

- structure of switching network: $v=4, f=36$ AUs, $V=144$ AUs;

- structure of offered traffic:

- traffic classes: $m=3, t_{1}=1 \mathrm{AU}, \mu_{1}^{-1}=1$, $t_{2}=4$ AUs, $\mu_{2}^{-1}=1, t_{3}=10 \mathrm{AUs}, \mu_{3}^{-1}=1$;

- sets of traffic sources: $S=2, \mathbb{C}_{\mathrm{Er}, 1}=\{1,2,3\}$, $\eta_{\mathrm{Er}, 1,1}=0,7, \eta_{\mathrm{Er}, 1,2}=0,2, \eta_{\mathrm{Er}, 1,3}=0,1$, $\mathbb{C}_{\mathrm{En}, 2}=\{1,2,3\}, \eta_{\mathrm{En}, 2,1}=0,6, \eta_{\mathrm{En}, 2,2}=0,2$, $\eta_{\mathrm{En}, 2,3}=0,2, N_{\mathrm{En}, 2}=900$,

- threshold mechanism: $R_{1}=R_{2}=119$ AUs.

\section{- System 4-Bandwidth reservation mechanism:}

- structure of switching network: $v=4, f=34$ AUs, $V=136 \mathrm{AUs}$

- structure of offered traffic:

- traffic classes: $m=4, t_{1}=1$ AUs, $\mu_{1}^{-1}=1$, $t_{2}=4$ AUs, $\mu_{2}^{-1}=1, t_{3}=6$ AUs, $\mu_{3}^{-1}=1$, $t_{4}=10 \mathrm{AUs}, \mu_{4}^{-1}=1$;

- sets of traffic sources: $S=2, \mathbb{C}_{\mathrm{Pa}, 1}=\{1,2,4\}$, $\eta_{\mathrm{Pa}, 1,1}=0,7, \eta_{\mathrm{Pa}, 1,2}=0,2, \eta_{\mathrm{Pa}, 1,4}=0,1$, $S_{\mathrm{Pa}, 1}=900, \mathbb{C}_{\mathrm{En}, 2}=\{1,3,4\}, \eta_{\mathrm{En}, 2,1}=0,6$, $\eta_{\mathrm{En}, 2,3}=0,2, \eta_{\mathrm{En}, 2,4}=0,2, N_{\mathrm{En}, 2}=900$; 
Fig. 6 Point-to-group blocking probability in System 1-PGPPBRec-U method

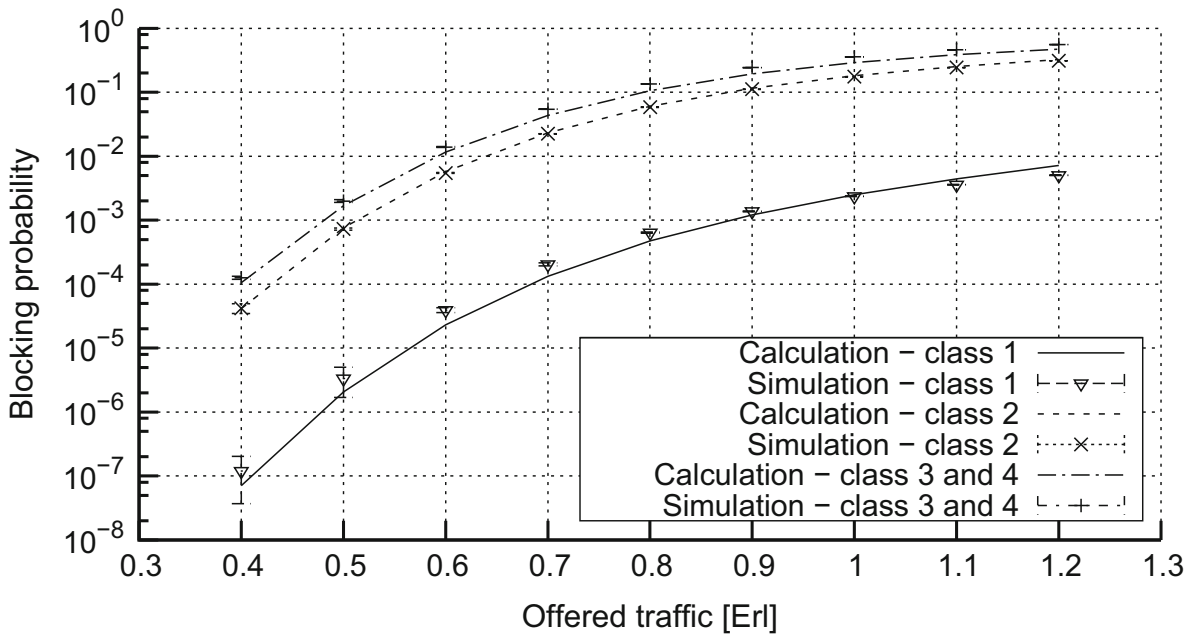

Fig. 7 Point-to-point blocking probability in System 2-PGPPBRec-U method

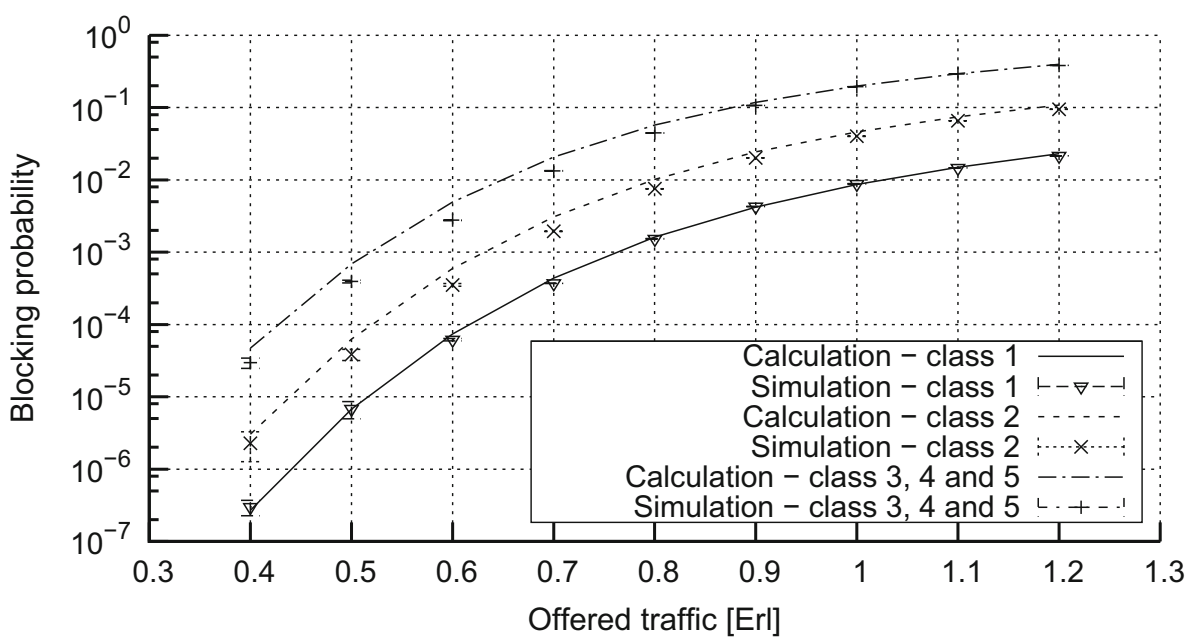

- threshold mechanism: $R_{1}=R_{2}=R_{3}=112$ AUs.

The results of the calculations and simulations are presented as the function of unitary traffic $a$ offered to a single AU of the system:

$$
\begin{aligned}
a= & {\left[\sum_{i=1}^{s_{I}} \lambda_{\mathrm{Er}, i}\left(\sum_{c=1}^{c_{\mathrm{Er}, i}} t_{c, 0} \eta_{\mathrm{Er}, i, c}\right)\left(\sum_{c=1}^{c_{\mathrm{Er}, i}} \eta_{\mathrm{Er}, i, c} / \mu_{c}\right)\right.} \\
& +\sum_{j=1}^{s_{J}} \gamma_{\mathrm{En}, j} N_{\mathrm{En}, j}\left(\sum_{c=1}^{c_{\mathrm{En}, j}} t_{c, 0} \eta_{\mathrm{En}, j, c}\right)\left(\sum_{c=1}^{c_{\mathrm{En}, j}} \eta_{\mathrm{En}, j, c} / \mu_{c}\right) \\
& +\sum_{k=1}^{s_{K}} \gamma_{\mathrm{Pa}, k} S_{\mathrm{Pa}, k} \\
& \left.\left(\sum_{c=1}^{c_{\mathrm{Pa}, k}} t_{c, 0} \eta_{\mathrm{Pa}, k, c}\right)\left(\sum_{c=1}^{c_{\mathrm{Pa}, k}} \eta_{\mathrm{Pa}, k, c} / \mu_{c}\right)\right] /(v v f) .
\end{aligned}
$$

The first system to be examined was a switching network with multiservice traffic sources and threshold mechanisms, defined as System 1 and System 2. The obtained results for point-to-group blocking probability for System 1 and pointto-point blocking probability for System 2 are presented in Figs. 6 and 7, respectively

The subsequent investigations dealt with an examination of the accuracy of the proposed method in determining the blocking probability in switching networks with reservation mechanisms. Figure 8 shows the results for the point-togroup blocking probability obtained for System 3. The reservation algorithms ensure a substantial decrease in the blocking probabilities of traffic classes for which the reservation mechanism has been introduced. In the particular cases, the mechanism can be applied to equalise the level of call service of selected or all traffic streams, which can be observed in Fig. 8. It is possible by changing the values of the reservation threshold.

Additionally, a study designed to compare the accuracy of the PGB-R method (developed in [50]) and the PGPPBRecU method (Fig. 9) was carried out. A switching network with the structure of System 4 was used for the purpose. Figure 9 
Fig. 8 Point-to-group blocking probability in System

3-PGPPBRec-U method
Fig. 9 Point-to-group blocking probability in System 4 - class 4

Fig. 10 Point-to-group blocking probability in System 1 —class 3 and 4
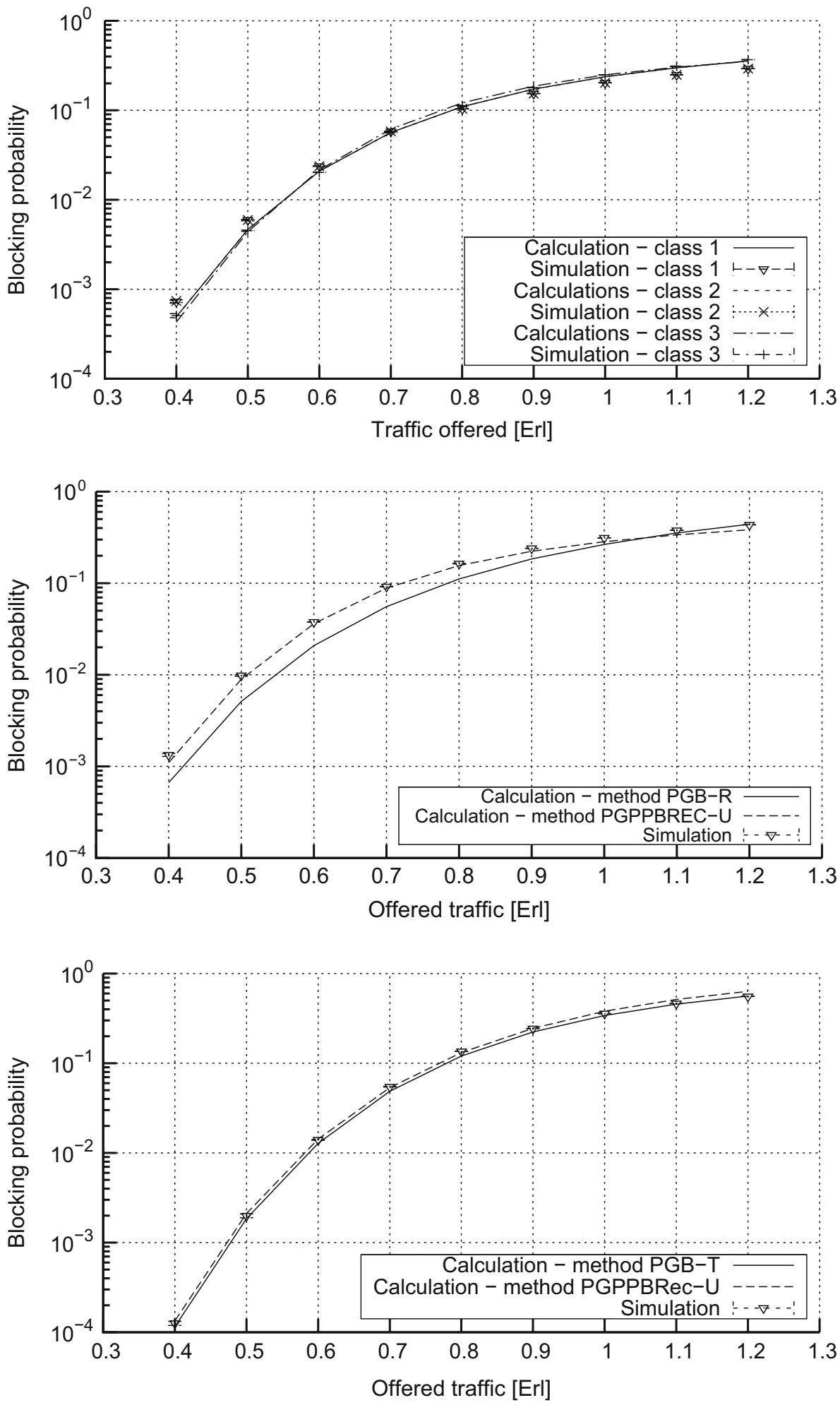

presents the results obtained for the fourth class of offered traffic.

With the analysis of the studied switching networks with multiservice traffic sources and threshold mechanisms it can be stated that the proposed method of determining point- to-group blocking probability and point-to-point blocking probability is characterised by high accuracy. In the case of networks with threshold mechanisms, the results obtained on the basis of generalised methods are similar to the results obtained on the basis of the corresponding method in [51]. 
Figure 10 presents a comparison of the blocking probabilities calculated using both the generalised method and the method presented in [51]. The application of the generalised recurrent PGPPBRec-U method ensures higher accuracy in the case of systems with reservation.

\section{Summary}

This article proposes the new generalised method of determining the occupancy distribution and total blocking probability in multi-stage switching networks in which resource allocation control mechanisms for calls of particular traffic classes of multiservice traffic (generated by multiservice sources) are introduced depending on the occupancy state of output directions of the switching network.

In the method of modelling switching networks, two generalisations have been introduced. Firstly, this method allows the modelling of networks with both point-to-group and point-to-point selection to be simultaneously modelled. Secondly, the developed method enables making the way the occupancy distribution and blocking probability in interstage links is determined independent of the type of the applied mechanism in output directions, i.e. resource reservation or threshold mechanism. The approach presented in the article makes it possible to apply a model of the full-availability group without threshold mechanisms and reservation mechanism in modelling inter-stage links.

The accuracy of the proposed method for modelling switching networks was established as a result of a comparison of the obtained analytical results with simulation data, both for reservation mechanisms and threshold mechanisms. In addition, the results obtained on the basis of the developed generalised method were compared with the results obtained on the basis of specialised methods, developed separately for each type of selection (point-to-point and point-to-group) and both types of resource allocation mechanisms for calls (resource reservation and threshold mechanisms). Numerous studies on the subject, some presented in this article, clearly indicate that the developed method ensures a similar (never worse) level of accuracy compared to specialised methods of modelling networks with the threshold mechanism and far higher accuracy in the case of networks with resource reservation.

Acknowledgements Funding was provided by Ministerstwo Nauki i Szkolnictwa Wyższego (Grant No. 08/82/DSPB/8216).

Open Access This article is distributed under the terms of the Creative Commons Attribution 4.0 International License (http://creativecomm ons.org/licenses/by/4.0/), which permits unrestricted use, distribution, and reproduction in any medium, provided you give appropriate credit to the original author(s) and the source, provide a link to the Creative Commons license, and indicate if changes were made.

\section{References}

1. Moscholios, I., Logothetis, M., \& Boucouvalas, A. (2015). Blocking probabilities of elastic and adaptive calls in the Erlang multirate loss model under the threshold policy. Telecommunication Systems, 62, 245-262.

2. Imran, M., Landais, P., Collier, M., \& Katrinis, K. (2015). Performance analysis of optical burst switching with fast optical switches for data center networks. In 2015 17th International conference on transparent optical networks (ICTON) (pp. 1-4).

3. Gauger, C., Kühn, P., Breusegem, E., Pickavet, M., \& Demeester, P. (2006). Hybrid optical network architectures: Bringing packets and circuits together. IEEE Communications Magazine, 44, 36-42.

4. Napoli, A., \& Lord, A. (2016). Elastic optical networks: Introduction. Journal of Optical Communications and Networking, 8, EON1-EON1.

5. Klonidis, D., Zakynthinos, P., \& Tomkos, I. (2015). Opportunities and challenges in the network planning of spatially and spectrally elastic optical networks. In 2015 17th International conference on transparent optical networks (ICTON) (pp. 1-4).

6. Chrysos, N., Minkenberg, C., Rudquist, M., Basso, C., \& Vanderpool, B. (2015). Scoc: High-radix switches made of bufferless Clos networks. In 2015 IEEE 21 st International symposium on high performance computer architecture (HPCA) (pp. 402-414).

7. Xia, Y., Hamdi, M., \& Chao, H. (2016). A practical largecapacity three-stage buffered Clos-network switch architecture. IEEE Transactions on Parallel and Distributed Systems, 27, 317328 .

8. Ye, T., Lee, T., \& Hu, W. (2015). Awg-based non-blocking Clos networks. IEEE/ACM Transactions on Networking, 23, 491-504.

9. Zhang, M., Qiu, Z., \& Gao, Y. (2014). Space-memory-memory Clos-network switches with in-sequence service. IET Communications, 8, 2825-2833.

10. Zal, M., \& Wojtysiak, P. (2014). An energy-efficient control algorithms for switching fabrics. In 2014 16th International on telecommunications network strategy and planning symposium (networks) (pp. 1-5).

11. McDermott, T., \& Brewer, T. (2003). Large-scale IP router using a high-speed optical switch element. Journal of Optical Networking, 2, 229-240.

12. Liboiron-Ladouceur, O., \& Bergman, K. (2007). Optimization of a switching node for optical multistage interconnection networks. IEEE Photonics Technology Letters, 19, 1658-1660.

13. Sehery, W., \& Clancy, T. (2015). Load balancing in data center networks with folded-Clos architectures. In 2015 1st IEEE conference on network softwarization (NetSoft) (pp. 1-6).

14. Liew, S. C., Ng, M.-H., \& Chan, C. W. (1998). Blocking and nonblocking multirate Clos switching networks. IEEE/ACM Transactions on Networking, 6, 307-318.

15. Ye, T., Lee, T. T., \& Hu, W. (2014). Awg-based non-blocking Clos networks. IEEE Transactions on Communications, 23, 491-504.

16. Danilewicz, G., \& Rajewski, R. (2014). The architecture and strictsense nonblocking conditions of a new baseline-based optical switching network composed of symmetrical and asymmetrical switching elements. IEEE Transactions on Communications, 62, 1058-1069.

17. Rajewski, R. (2014). The rearrangeable nonblocking conditions in the multi-MBA (n, e, 2) switching network. In 2014 16th International telecommunications network strategy and planning symposium (networks) (pp. 1-6).

18. Pras, A., Nieuwenhuis, L., van de Meent, R., \& Mandjes, M. (2009). Dimensioning network links: A new look at equivalent bandwidth. IEEE Network, 23, 5-10.

19. Bonald, T. (2009). A recursive formula for estimating the packet loss rate in IP networks. In Proceedings of the fourth international 
ICST conference on performance evaluation methodologies and tools, VALUETOOLS '09 (pp. 56:1-56:2). Brussels: ICST (Institute for Computer Sciences, Social-Informatics and Telecommunications Engineering).

20. Katangur, A. K., Akkaladevi, S., \& Pan, Y. (2007). Analyzing the performance of optical multistage interconnection networks with limited crosstalk. Cluster Computing, 10, 241-250.

21. Kaczmarek, S. (2002). Multistage optical switching networks. Journal of Telecommunications and Information Technology, 2, $21-25$.

22. Hanczewski, S., Stasiak, M., \& Zwierzykowski, P. (2015). Modelling of the access part of a multi-service mobile network with service priorities. EURASIP Journal on Wireless Communications and Networking, 2015, 1-14.

23. Zhang, H., Nie, Y., Cheng, J., Leung, V. C. M., \& Nallanathan, A. (2017). Sensing time optimization and power control for energy efficient cognitive small cell with imperfect hybrid spectrum sensing. IEEE Transactions on Wireless Communications, 16, 730 743.

24. Mashaly, M., \& Kühn, P. J. (2012). Load balancing in cloudbased content delivery networks using adaptive server activation/deactivation. In Proceedings of the 24th international teletraffic congress, ITC '12 (pp. 21:1-21:3). International Teletraffic Congress.

25. Głąbowski, M., Stasiak, M., \& Weissenberg, J. (2012). Properties of recurrent equations for the full-availability group with BPP traffic. Mathematical Problems in Engineering. doi:10.1155/2012/ 547909.

26. Conradt, J., \& Buchheister, A. (1985). Considerations on loss probability of multi-slot connections. In Proceedings of 11th international teletraffic congress, Kyoto (pp. 4.4B-2.1).

27. Kaufman, J. (1981). Blocking in a shared resource environment. IEEE Transactions on Communications, 29, 1474-1481.

28. Roberts, J. (1981). A service system with heterogeneous user requirements-Application to multi-service telecommunications systems. In G. Pujolle (Ed.), Proceedings of performance of data communications systems and their applications (pp. 423-431). Amsterdam: North Holland.

29. Weber, A., Fischer, W., \& Huber, M. (1991). Multichannel circuit switching-Performance evaluation of switching networks. IEEE Journal on Selected Areas in Communications, 9, 226-232.

30. Jacobaeus, C. (1950). A study on congestion in link-systems. Ericsson Technics, 48, 1-68.

31. Rigault, C. (2002). Clos networks: A correction of the Jacobaeus result. Annales des Télécommunications, 57, 1244-1252.

32. Stasiak, M. (1996). Combinatorial considerations for switching systems carrying multi-channel traffic streams. Annales des Télécommunications, 51, 611-625.

33. Charkiewicz, A. (1959). An approximate method for calculating the number of junctions in a crossbar system exchange. Elektrosvyaz, $2,55-63$.

34. Lotze, A., Roder, A., \& Thierer, G. (1976). Point-to-point loss in case of multiple marking attempts. In Proceedings of 8 th international teletraffic congress, Melbourne, supplement to paper $547 / 1-44$

35. Rothmaier, K., \& Scheller, R. (1981). Design of economic PCM arrays with a prescribed grade of service. IEEE Transactions on Communications, 29, 925-935.

36. Ershov, V. (1984). Some further studies on effective accessibility: Fundamentals of teletraffic theory. In Proceedings of 3rd international seminar on teletraffic theory, Moscow (pp. 193-196).

37. Fitzpatrick, G., Beshai, M., \& Munter, E. (1991). Analysis of largescale three-stage networks serving multirate traffic. In Proceedings of 13th international teletraffic congress, Copenhagen (Vol. 14, pp. 905-910).
38. Głą bowski, M., \& Stasiak, M. D. (2014). Modelling of multiservice switching networks with overflow links for any traffic class. IET Circuits, Devices \& Systems, 8, 358-366.

39. Hanczewski, S., Sobieraj, M., \& Stasiak, M. D. (2016). The direct method of effective availability for switching networks with multiservice traffic. IEICE Transactions on Communications, E99-B, 1291-1301.

40. Głąbowski, M., \& Stasiak, M. (2002). Point-to-point blocking probability in switching networks with reservation. Annals of Telecommunications, 57, 798-831.

41. Głąbowski, M., \& Sobieraj, M. (2009). Point-to-group blocking probability in switching networks with threshold mechanisms. In Proceedings of the fifth advanced international conference on telecommunications, Venezia (pp. 95-100). IEEE Computer Society.

42. Stasiak, M., \& Zwierzykowski, P. (2002). Point-to-group blocking in the switching networks with unicast and multicast switching. Performance Evaluation, 48, 249-267.

43. Ross, K. (1995). Multiservice loss models for broadband telecommunication network. Berlin: Springer.

44. Moscholios, I. D., Logothetis, M. D., Vardakas, J. S., \& Boucouvalas, A. C. (2015). Congestion probabilities of elastic and adaptive calls in Erlang-Engset multirate loss models under the threshold and bandwidth reservation policies. Computer Networks, 92(Part 1), 1-23.

45. Stamatelos, G., \& Hayes, J. (1994). Admission-control technics with application to broadband networks. Computer Communication, 17, 663-673.

46. Głąbowski, M., Sobieraj, M., \& Stasiak, M. (2012). Modeling switching networks with multi-service sources and point-to-group selection. In Proceedings of the 18th Asia-Pacific conference on communications (APCC 2012), Jeju Island, Korea (pp. 686-691).

47. Głąbowski, M. (2007). Blocking probability in multi-service switching networks with finite source population. In Proceedings of 2007 IEEE international conference on telecommunications, Penang (pp. 793-798).

48. Głą bowski, M. (2008). Recurrent method for blocking probability calculation in multi-service switching networks with BPP traffic. In $\mathrm{N}$. Thomas \& C. Juiz (Eds.), Computer performance engineering. Lecture notes in computer science (Vol. 5261, pp. 152-167). Berlin: Springer.

49. Głąbowski, M., Sobieraj, M., \& Stasiak, M. (2011). Point-to-point blocking probability in switching networks with hysteresis. In G. Danilewicz, M. Głą bowski, P. J. Kühn, \& M. Pióro (Eds.), Proceedings of the first European teletraffic seminar: An initiative to strengthen collaboration on network design and performance. Poznan: Poznan University of Technology.

50. Głąbowski, M., \& Sobieraj, M. (2014). Point-to-group blocking probability in switching networks with multi-service sources and bandwidth reservation. In 2014 9th International symposium on communication systems, networks digital signal processing (CSNDSP) (pp. 93-98).

51. Głąbowski, M., \& Sobieraj, M. (2014). Modelling of network nodes with threshold mechanisms and multi-service sources. In 2014 16th International on telecommunications network strategy and planning symposium (networks) (pp. 1-7).

52. Stasiak, M., Głąbowski, M., Wiśniewski, A., \& Zwierzykowski, P. (2011). Modeling and dimensioning of mobile networks. New York: Wiley.

53. Akimaru, H., \& Kawashima, K. (1999). Teletraffic: Theory and application. Berlin: Springer.

54. Głąbowski, M., \& Stasiak, M. (2004). Multi-rate model of the group of separated transmission links of various capacities. In J. de Souza, P. Dini, \& P. Lorenz (Eds.), Telecommunications and networking-ICT 2004. Lecture notes in computer science (Vol. 3124, pp. 1101-1106). Berlin: Springer. 
55. Głąbowski, M., Kaliszan, A., \& Stasiak, M. (2010). Modeling product-form state-dependent systems with BPP traffic. Performance Evaluation, 67, 174-197.

56. Stasiak, M. (1993). Blocking probability in a limited-availability group carrying mixture of different multichannel traffic streams. Annales des Télécommunications, 48, 71-76.

57. Głąbowski, M., Hanczewski, S., Stasiak, M., \& Weissenberg, J. (2012). Modeling Erlang's Ideal Grading with multi-rate BPP traffic. Mathematical Problems in Engineering. doi:10.1155/2012/ 456910.

58. Stasiak, M. (1993). An approximate model of a switching network carrying mixture of different multichannel traffic streams. IEEE Transactions on Communications, 41, 836-840.

59. Lee, C. (1955). Analysis of switching networks. Bell System Technical Journal, 34, 1287-1315.

60. Głąbowski, M. (2006). Recurrent calculation of blocking probability in multiservice switching networks. In Proceedings of Asia-Pacific conference on communications, Busan (pp. 1-5).

61. Lotze, A., Roder, A., \& Thierer, G. (1976). Point-to-point selection versus point-to-group selection in link systems. In Proceedings of 8th international teletraffic congress, Melbourne (pp. 541/1$541 / 5)$.

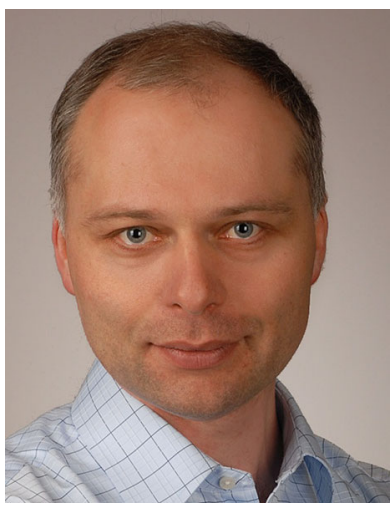

Mariusz Głąbowski received the M.Sc., Ph.D. and D.Sc. (Habilitation) degrees in telecommunication from the Poznan University of Technology, Poland, in 1997, 2001, and 2010, respectively. Since 1997 he has been working in the Department of Electronics and Telecommunications, Poznan University of Technology. He is engaged in research and teaching in the area of performance analysis and modeling of multiservice networks and switching systems. He is the author/co-author of 4 books, 7 book chapters and of over 100 papers which have been published in communication journals and presented at national and international conferences. He has refereed articles for many international conferences and magazines, including: IEEE Globecom, IEEE ICC, IEEE HPRS, IEEE Transactions on Communications, IEEE Communications Magazine, Computer Networks, IEEE Communications Letters, IEEE Transactions on Wireless Communications, Performance Evaluation, European Transactions on Telecommunications.

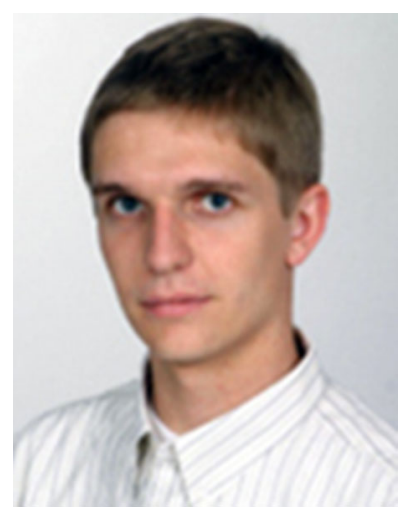

Maciej Sobieraj received M.Sc. and Ph.D. degrees in Electronics and Telecommunications from Poznan University of Technology, Poland, in 2008 and 2014, respectively Science 2007 he has been working at the Chair of Communication and Computer Networks at the Faculty of Electronics and Telecommunications at Poznan University of Technology. He is the co-author of more than 40 scientific papers. $\mathrm{He}$ is engaged in research in the area of modeling multiservice cellular systems and switching networks and traffic engineering in TCP/IP networks. 